\title{
An experimental study of airfoil instability tonal noise with trailing edge serrations
}

\author{
Tze Pei Chong ${ }^{a} *$, Phillip Joseph ${ }^{b}$ \\ ${ }^{a}$ School of Engineering and Design, Brunel University, Uxbridge, UB8 3PH,UK \\ ${ }^{\mathrm{b}}$ Institute of Sound and Vibration Research, University of Southampton, Southampton, SO17 1BJ, UK
}

\begin{abstract}
This paper presents an experimental study of the effect of trailing edge serrations on airfoil instability noise. Detailed aeroacoustic measurements are presented of the noise radiated by an NACA-0012 airfoil with trailing edge serrations in a low to moderate speed flow under acoustical free field conditions. The existence of a separated boundary layer near the trailing edge of the airfoil at an angle of attack of 4.2 degree has been experimentally identified by a surface mounted hot-film arrays technique. Hot-wire results have shown that the saw-tooth surface can trigger a bypass transition and prevent the boundary layer from becoming separated. Without the separated boundary layer to act as an amplifier for the incoming Tollmien-Schlichting waves, the intensity and spectral characteristic of the radiated tonal noise can be affected depending upon the serration geometry. Particle Imaging Velocimetry (PIV) measurements of the airfoil wakes for a straight and serrated trailing edge are also reported in this paper. These measurements show that localized normal-component velocity fluctuations that are present in a small region of the wake from the laminar airfoil become weakened once serrations are introduced. Owing to the above unique characteristics of the serrated trailing edges, we are able to further investigate the mechanisms of airfoil instability tonal noise with special emphasis on the assessment of the wake and non-wake based aeroacoustic feedback model. It has been shown that the instability tonal noise generated at an angle of attack below approximately one degree could involve several complex mechanisms. On the other hand, the non-wake based aeroacoustic feedback mechanism alone is sufficient to predict all discrete tone frequencies accurately when the airfoil is at a moderate angle of attack.
\end{abstract}

Keywords: Airfoil self-noise, trailing edge serrations, aeroacoustic feedback loops, tone noise

\footnotetext{
* Corresponding author. Tel.: + 441895266370

E-mail address: t.p.chong@brunel.ac.uk
} 


\section{Introduction}

Research into the characteristics and mechanisms of airfoil trailing edge self-noise has continued for more than fifty years. This prolonged period of research effort has arisen from the need to understand, predict and control the self-noise from airfoils located within aero-engines, wind turbines, high lift devices on aircraft airframes, as well as the noise from many common aerodynamic noise sources such as ventilation fans.

The character and level of trailing edge self-noise are known to be highly sensitive to Reynolds number, angle of attack, airfoil geometry and trailing edge bluntness [1]. Trailing edge noise radiated in high Reynolds number flow $\left(>10^{6}\right)$, for example, is typically broadband in nature. By contrast, for low Reynolds number with minimal residue turbulence in the freestream, trailing edge noise has a distinctive narrowband character comprising a broadband hump superimposed with a number of high amplitude "tones" [2, 3]. In this situation the boundary layer on the airfoil surface is mainly laminar but potentially unstable. Under a certain range of conditions, hydrodynamic instabilities such as the Tollmien-Schlichting (T-S) waves, grow in the boundary layer and eventually scatter into noise at the trailing edge. This mechanism of self-noise is referred to as "instability tonal noise" and is the subject of investigation in this paper.

The scattering of T-S waves at the trailing edge also gives rise to a "broadband hump" in the radiation spectrum. This mechanism is supported by a number of studies in which the frequencies of the maximum T-S wave amplification occur at frequencies that closely coincide with the frequencies of the main broadband hump [3-5]. Tam [6] has proposed that the scattering process at the trailing edge will form part of a closed aeroacoustic feedback loop, which is responsible for the generation of the multiple, high amplitude "tones". Since then a number of models have been proposed in the literature to explain this feedback loop. Some of these are illustrated in Fig. 1. Model A $[2,5]$ assumes that acoustic waves generated at the trailing edge will radiate upstream to disturb the boundary layer from which the T-S wave originated. Subsequently the T-S waves propagate to the trailing edge and radiate the broadband hump, and so on. In Model B [7], the feedback loops consist of a dipole noise source in the wake and the upstream inception points of the T-S wave instabilities on both the airfoil's pressure and suction surfaces. In Model C [6], the feedback loop is between the noise source in the wake and the trailing edge. More recently, Tam and Ju [8] in their DNS study have observed that the energy source for the airfoil tones is the interaction between the near wake oscillation driven by a Kelvin-Helmholtz instability and the trailing edge of the airfoil. Most of these models propose that the tones occur at frequencies for which the total phase change around the loop equals $2 \pi n$, where $n$ is a positive integer. 
Of central importance in this study is the priori assumption that acoustic scattering of T-S waves is only effective, and hence noise is radiated efficiently, when the boundary layer along the airfoil separates. Considerable work on a NACA-0012 airfoil has shown that the pre-requisite condition for a broadband hump and/or tones to occur is the existence of a separation region near the trailing edge on the pressure surface $[4,9,10]$. It was concluded that the incoming T-S waves must be amplified by the separating shear layer before tonal noise can be radiated effectively. The intensity of the tones is also found to be highly sensitive to the bubble length [9]. For most symmetrical airfoils, an adverse pressure gradient always prevails at the aft region of the airfoil, and its level depends on the airfoil's profile and angle of attack. These factors in turn influence the separation region, which ultimately affects the intensity and frequency of the radiated instability tonal noise.

To date, the study of trailing edge noise of airfoils with serrations has been almost exclusively related to the reduction of the broadband self-noise [11-14]. As mentioned previously, broadband self-noise is mostly associated with turbulent boundary layers at high Reynolds numbers. On the other hand, it has been reported that the introduction of saw-tooth trailing edges can reduce the tendency of flow separation in an adverse pressure gradient region [15]. In low Reynolds number flow, the laminar boundary layer near the saw-tooth trailing edge will therefore be more resistant to separation, which could directly affect the efficiency of the instability noise radiation. The use of serrations is therefore potentially very effective in controlling instability tonal tones. However, it would appear that their use in this application has not previously been addressed in the literature.

It is important to recognize that the use of serrations to reduce instability self-noise and broadband self-noise involves different mechanisms. This paper focuses solely on the former and relates to an airfoil noise study performed at low to medium Reynolds number flows where, for most of the cases considered, no boundary layer transition occurred on the pressure surface of the straight trailing edge. The existence of a separated boundary layer near an airfoil's trailing edge has been considered as one of several pre-requisites needed to produce tonal noise. The paper aims to investigate, through the use of trailing edge serrations, whether flow separation could be prevented which then provides reductions in the instability tonal noise. Owing to the ability to produce turbulent wake flow by a serrated trailing edge, which could potentially eliminate any tonal noise source in the wake as per the feedback Models B and C, the second objective of this paper is to provide more insight into the different aeroacoustic feedback mechanisms. The third objective is to establish the relationship between the serration parameters (in terms of the serration angle and root-to-tip length) and the level of instability self-noise reduction. 


\section{Airfoil design and experimental setups}

\subsection{Airfoil design}

A NACA-0012 airfoil with $0.15 \mathrm{~m}$ chord and $0.45 \mathrm{~m}$ span was used in this study. The airfoil consists of changeable trailing edges to allow the investigation of different saw-tooth patterns (including a straight trailing edge). As shown in Fig. 2, the main geometrical parameters associated with a sawtooth serration are the serration angle $(\varepsilon)$, tip-to-root distance $(2 h)$ and serration wavelength $(\lambda)$. The figure also includes a table that summarizes the parameters associated with the four saw-tooth geometries tested in this study (S1, S2, S2" and S3). Note that $X_{\text {root }}$ represents the location of the root of the saw-tooth, which is expressed as a fraction of the airfoil chord, $C$. Although certain degrees of bluntness are present at the $X_{\text {root }}$ for the serrated trailing edges, no bluntness-induced vortex shedding tonal noise is produced in our test cases. A Cartesian coordinate system, as shown in Fig. 3, is used to describe the spatial distribution of the boundary layer on the airfoil's surfaces. The $x, y$ and $z$ directions represent the streamwise, transverse (wall-normal) and spanwise directions respectively. Note that $x=0$ is situated at the leading edge and $y=0$ occurs at the wall surface.

\subsection{Experimental setup for the ISVR open jet wind tunnel}

\subsubsection{Free field noise measurement}

Free field acoustic measurements of airfoil self-noise were conducted in the open jet wind tunnel in the ISVR, at the University of Southampton in the UK. The acoustic tunnel is situated in an $8 \mathrm{~m} \times 8$ $\mathrm{m} \times 8 \mathrm{~m}$ anechoic chamber. The nozzle exit is a rectangular section with a dimension of $0.15 \mathrm{~m} \times 0.45$ $\mathrm{m}$. This acoustic tunnel can achieve turbulence intensities as low as 0.1 percent at Mach number up to 0.3 , while maintaining low background noise $[16,17]$.

Figure 3 shows a schematic of the experimental setup for the free field noise measurements. The airfoil under investigation here was held by two side plates attached to the nozzle lips. The range of jet speeds, $v_{\mathrm{j}}$, under investigation here was $10-60 \mathrm{~ms}^{-1}$, which corresponds to Reynolds numbers based on the airfoil chord length of between $1 \times 10^{5}$ and $6 \times 10^{5}$ respectively. Because of the finite size of the open jet nozzle, the airfoil which is set at a "geometrical" angle of attack, $\theta_{\mathrm{g}}$, relative to the jet axis will inevitably induce flow curvature and downwash deflection of the incident flow, which could affect the pressure gradient distribution and the lift force. To correct this type of installation effect a two-dimensional open wind tunnel correction method [1] was used to calculate the "effective" angle of attack, $\theta$, from the "geometrical" angle $\theta_{\mathrm{g}}$. The airfoil instability tonal noise 
was measured at a total of three geometrical angles, $\theta_{\mathrm{g}}=0^{\circ}, 5^{\circ}$ and $15^{\circ}$. However, for brevity this paper will only present the results pertaining to the $\theta_{\mathrm{g}}=15^{\circ}$ case, which corresponds to $\theta=4.2^{\circ}$ after applying the wind tunnel correction. The reader can refer to our earlier paper in Chong et al. [18] for the other cases, i.e. $\theta_{\mathrm{g}}=0^{\circ}$ and $5^{\circ}$ with regard to the characteristics of the instability tonal noise when the airfoil is subjected to trailing edge serrations.

The far field noise measurements were made by a $1 / 2$ inch Brüel \& Kjær microphone (Type 4191) situated at $1.25 \mathrm{~m}$ from the airfoil trailing edge at a polar angle of $\zeta=90^{\circ}$. This microphone is mounted in the mid-span plane of the airfoil model. Noise data was sampled at $30 \mathrm{kHz}$ for 13.33 seconds using a 24-bit National Instrument analogue-to-digital (A/D) card. The digitized data was low-pass filtered at more than half of the sampling rate to avoid signal aliasing. The data was then windowed (with a size of 4096 points) and averaged to obtain the Power Spectral Density, PSD.

\subsubsection{Flow measurement using surface mounted hot-film arrays and a single hot-wire}

It is important to be able to determine the location of boundary layer separation, if it exists, before we can firmly establish its causal link with the T-S waves, the radiated instability tonal noise and the serrated trailing edge as per the current paper's objectives. Experimental techniques, such as Laser Doppler Anemometry (LDA), surface visualization, pressure transducers and non-intrusive surface mounted hot-film arrays, have been proven to be effective in identifying laminar separation. With regard to the surface mounted hot-film arrays in which the sensors are operated simultaneously, the point of laminar separation or flow reversal is identified by a $180^{\circ}$ phase shift between velocity sensors located in the separation where flow bifurcation occurs [19-21]. Surface mounted hot-film arrays were employed in the present study to identify the separation point near the airfoil's pressure surface trailing edge. However, it should be noted that the $180^{\circ}$ phase shift technique is only applicable for flows where the main flow disturbance is non-convective in nature (e.g. a leading edge separation bubble). When a convective disturbance is present (e.g. T-S waves) in the neighbourhood of a separation region, a slightly different interpretation is needed. This will be discussed in Section 4.2.

The surface mounted hot-films used in this study, which have an overall thickness of $50 \mu \mathrm{m}$, are manufactured by DANTEC of type 55R47. The sensing element is $0.9 \mathrm{~mm} \times 0.1 \mathrm{~mm}$ in size and is connected to gold-plated leads. During the measurement an overheat ratio of 1.3 was used for the hot-films. The choice of such a relative low overheat ratio is a compromise between a reasonable velocity sensitivity and a minimal thermal destabilization to the otherwise isothermal boundary 
layer. Figure 4 shows the arrangement of the five surface mounted hot-film arrays (labelled HF1, HF2 and so on) near the pressure surface of the NACA-0012 airfoil with a straight SO trailing edge. Note that each of the sensing elements of the hot-film sensors is separated by an equidistance of $6 \mathrm{~mm}$ in the longitudinal direction. The sensing elements of HF1, HF2, HF3, HF4 and HF5 are located at $x / C=$ $0.79,0.83,0.87,0.91$ and 0.95 respectively. Note that the sensing element of HF3 also coincides with the root of the serrated trailing edges, $X_{\text {root }}$, for the S1, S2 and S3 cases. Unlike some of the custommade surface mounted hot-film arrays where the conductive leads are extended significantly away from the measurement area [19-21], the DANTEC hot-film sensors have short conductive leads which mean that the electrical connection between the gold-plated leads and the copper wires has to be made quite close to the measurement area. In order to avoid flow interference in the longitudinal direction, an $8 \mathrm{~mm}$ spanwise offset was introduced for each successive sensor. This arrangement will not significantly affect the spatial resolution and coherence of the signals due to the predominantly two-dimensional nature of the laminar separation as well as the T-S waves. The signals from the five hot-film sensors were sampled simultaneously at $44.1 \mathrm{kHz}$ for about 13.33 seconds by a 24-bit National Instrument A/D card. The digitized data was low-pass filtered at more than half of the sampling frequency.

Because of the physical size of the surface mounted hot-film sensors, they are not suitable to be used for the serrated trailing edges due to the presence of gaps between the saw-teeth. In order to examine the state of the boundary layer on the serrated surface, a single hot-wire probe $(5 \mu \mathrm{m}$ diameter DANTEC 55P14) was used to measure the mean and fluctuating velocities at an overheat ratio of 1.8. Signals from the hot-wire were digitized by a 12-bit A/D converter at a sampling frequency of $5 \mathrm{kHz}$, after low-pass filtered at $2.5 \mathrm{kHz}$, for 32,768 realizations. Note that the use of a different instrumentation system was due to the unavailability of the intended equipment during the time of experiment, which was performed in a separate wind tunnel. Nevertheless, the results obtained by the hot wire are still relevant for comparison.

\subsection{Experimental setup for the PIV measurement in a closed-section wind tunnel}

Aerodynamic measurements of the global velocity fields for the same airfoil model were conducted using the Particle Image Velocimetry (PIV) technique in an open-circuit, suction type wind tunnel with a $0.6 \mathrm{~m} \times 0.9 \mathrm{~m}$ working section. The airfoil model was mounted vertically in the working section, as shown in Fig. 5. The hydrodynamic development and noise radiation of the airfoil were 
measured at two angles of attack, $\theta=0^{\circ}$ and $2^{\circ}$. In this study only one freestream velocity of $15.9 \mathrm{~ms}^{-}$

${ }^{1}$ was investigated, which corresponds to Reynolds number of about $1.6 \times 10^{5}$.

The PIV system consists of a Nd:YAG laser (120 mJ at $532 \mathrm{~nm}$ ), a pulse generator and a CCD camera (spatial resolution $1289 \times 1024$ pixels). It was operated in double frame mode for image capturing. The frame rate of the CCD camera was set typically at 2 frames per second. The particle tracing elements produced by the smoke generator in this study were about $2 \mu \mathrm{m}$ in diameter. It is important to ensure a uniform ingestion and spatial distribution of the smoke particles inside the working section when performing the PIV measurement. A procedure was developed whereby the entire laboratory was first filled with smoke. When the wind tunnel was switched on, the pressure difference between the laboratory and the working section allowed the smoke to be sucked uniformly into the working section through the contraction inlet. An advantage of this approach is that there is no need to place the smoke generator inside the wind tunnel. During the imageprocessing, an adaptive correlation analysis method with a minimum pixel-sized interrogation area of $16 \times 16$ and a $50 \% \times 50 \%$ overlap is used to improve the resolution of the velocity vectors. To obtain good quality mean flow data, at least 300 instantaneous vector maps were measured for each case.

Despite the apparently high background noise of the wind tunnel, the amplitudes of the dominant tone frequencies are still above the background noise for freestream velocities at below $20 \mathrm{~ms}^{-1}$. To measure the radiated noise, a microphone was flush mounted to a square panel at the side of the working section. As shown in Fig. 5, the horizontal axis of the microphone is perpendicular to the trailing edge of the airfoil at the pressure surface. The acoustic data was also sampled at $30 \mathrm{kHz}$ for 13.33 seconds. The background noise of the facility, i.e. without an airfoil inside the working section, was measured prior to the commencement of the airfoil noise study. The data was then used to obtain the acoustic spectrum of the background noise, $\Phi_{b}(\omega)$, where $\omega$ is the frequency. Acoustic spectra measured with the airfoil mounted inside the tunnel are denoted by $\Phi(\omega)$.

\section{Linear stability analysis for the two-dimensional boundary layer instability}

Disturbances in the laminar boundary layer are assumed to be spatially growing two-dimensional T-S waves with fixed frequency and slowly changing complex wavenumber [4,5]. The wavenumber of the disturbances, $\alpha$, can be represented by: 


$$
\alpha=\alpha_{\mathrm{r}}+\mathrm{i} \alpha_{\mathrm{i}}
$$

where the subscripts $r$ and $i$ denote the real and imaginary parts, respectively. The stream function $\psi(x, y)$ associated with the T-S waves can be expressed by:

$$
\psi(x, y)=\phi(y) e^{\mathrm{i}\left(\int \alpha(x) \mathrm{d} x-\omega t\right)}
$$

where $\phi$ is the perturbation amplitude of the disturbances and $t$ is the time. The wavenumber of the least stable mode at any streamwise location $x$ on the airfoil surface can be obtained from solutions to the Orr-Sommerfeld equation:

$$
\phi^{i v}-2 \alpha^{2} \phi^{\prime \prime}+\alpha^{4} \phi=\mathrm{i} \operatorname{Re}_{\delta}\left[(\alpha U(y)-\omega)\left(\phi^{\prime \prime}-\alpha^{2} \phi\right)-\alpha U^{\prime \prime}(y) \phi\right]
$$

where $R e_{\delta}$ is the Reynolds number based on the boundary layer thickness, $U(y)$ is the boundary layer velocity profile at location $x$ on the airfoil surface and the primes on $\phi$ and $U(y)$ denote differentiation with respect to $y$. The required input parameter to solve Eq. (3) is the boundary layer velocity profile along the airfoil surface on the pressure surface. Since the boundary layer disturbances must varnish at the wall surface and the freestream, the following boundary conditions are applicable for $\phi$ :

$$
\begin{array}{ll}
\text { At } y=0, & \phi=\phi^{\prime}=0 \\
\text { As } y \rightarrow \infty, & \phi=\phi^{\prime}=0
\end{array}
$$

In this study, the boundary layer velocity profiles were obtained using a numerical method, which was originally developed and successfully implemented by Kingan and Pearse [5]. Calculation of the velocity profiles was made using the public domain code, XFOIL [22] to determine the shape factors and boundary layer displacement thicknesses over the airfoil surface. The Falkner-Skan velocity profile is well known for its accurate modelling of flow with rapidly changing pressure gradient, such as the flow around an airfoil. Because each Falkner-Skan velocity profile is associated with a unique shape factor, the boundary layer profile at each $x$ that matches the predicted shape factor can be solved. The Falkner-Skan profile is described by the following equation:

$$
f^{\prime \prime \prime}-f f^{\prime \prime}+\beta\left(1-f^{\prime 2}\right)=0
$$


where $f^{\prime}$ is the Falkner-Skan velocity profile, $\beta$ is the pressure gradient parameter and the prime denotes differentiation with respect to $y$. Because of the no-slip condition at the wall, and the finite thickness of the shear layer, the following boundary conditions apply:

$$
\begin{aligned}
& \text { At } y=0, \quad f=f^{\prime}=0 \\
& \text { As } y \rightarrow \infty, \quad f^{\prime} \rightarrow 1
\end{aligned}
$$

The Falkner-Skan equation was solved using the parallel shooting technique employing a $4^{\text {th }}$-order Runge-Kutta-Gill solver. This methodology has shown to be accurate when used to predict the velocity profile on the airfoil surface [5].

To ensure that this methodology can also be applied accurately in this work, a verification study was performed through hot-wire measurements of boundary layer velocity profiles at several $x$ locations near the trailing edge of the airfoil. A similarity variable, $\eta$ is introduced to represent the distance normal to the wall surface:

$$
\eta=\sqrt{\frac{1}{2-\beta}} \operatorname{Re}^{0.5} \frac{y}{x}
$$

where $\mathrm{Re}$ is the Reynolds number based on the streamwise distance $x$. Fig. 6 compares the measured and predicted velocity profiles at the airfoil's pressure surface for $x / C=0.80,0.87$ and 0.93 at $\theta=5^{\circ}$. The excellent overall agreement between the measurement and prediction confirms the robustness of the airfoil code for obtaining accurate prediction of the velocity profiles that are required by the boundary layer stability analysis described above.

Numerical solutions to the Orr-Sommerfeld equation in Eq. (3) were obtained using the Chebyshev matrix technique described by Kingan and Pearse [5]. The T-S wavenumber $\alpha$ of the least stable mode at a fixed frequency of the disturbances was solved at $x / C=0.02$ to 0.99 with a step size of 0.01 . The first point along the airfoil chord at which instability first appears, $x_{0}$, is identified with the location where $\operatorname{Im}\left\{\alpha_{i}\right\}<0$. The quantity $\alpha_{\mathrm{i}}$ can be regarded as the local T-S wave amplification rate pertinent to a particular $x / C$. It is also useful to calculate the total amplification factor, $A$, of the T-S wave, at any frequency, between $x / C=\varphi_{1}$ and $\varphi_{2}$, where $\varphi_{1}=x_{0} / C$. The equation is given by:

$$
A=e^{\left(-C \int_{\varphi_{1}}^{\varphi_{2}} \alpha_{\mathrm{i}}\left(\frac{x}{C}\right) \mathrm{d}\left(\frac{x}{C}\right)\right)}
$$


Note that $\varphi_{2}=0.99$ is applicable for a straight trailing edge. However the boundary layer instability should be scattered at the saw-tooth's root of a serrated trailing edge in which case $\varphi_{2}$ is equal to $X_{\text {root }}$ The validity of this postulation will be confirmed later in Section 4.3. It is also necessary to ensure that the serrated trailing edge does not alter the location of the rear flow stagnation point which could generate a very different global flow field if compared to the straight trailing edge case. A non-dimensional static pressure distribution along the airfoil is introduced:

$$
C_{\mathrm{p}}=\frac{P(x)-P_{\mathrm{s}}}{P_{\mathrm{o}}-P_{\mathrm{s}}}
$$

where $P(x)$ is the static pressure at a streamwise location of the airfoil. $P_{\mathrm{o}}$ and $P_{\mathrm{s}}$ are the total and static pressures of the freestream flow measured by a pitot-static tube. Figure 7a compares the axial distributions of $C_{p}$ along the suction surface at several angles of attacks for the S0 and S3 cases. The

$C_{p}$ values tend to decrease near the leading edge as the angle of attack increases from $0^{\circ}$ to $10^{\circ}$ due to the accelerated flow. The difference of the $C_{\mathrm{p}}$ values between the S0 and S3 cases are negligible. Similar results can also be obtained at the pressure surface, as shown in Fig. $7 \mathrm{~b}$. This suggests that the serration should not affect the pressure distributions for $x<X_{\text {root }}$. Therefore, any potential changes of the noise spectra and aerodynamic performances of an airfoil by adding the serrated geometry at the trailing edge can solely be attributed to the dynamics of the local flow within the saw-tooth region. This result confirms that the application of the two-dimensional instability analysis is still valid for an airfoil with a serrated trailing edge.

\section{Discussion of results}

\subsection{Airfoil instability noise with a straight trailing edge, SO (baseline case)}

Measurements of the airfoil self-noise radiation were made under free field condition in an anechoic chamber. This section presents the baseline results corresponding to the straight trailing edge, S0. A typical instability tonal noise spectrum is shown in Fig. 8. The spectrum exhibits a broadband hump with a center frequency occurring at $f_{s}$. A number of equally spaced discrete tones $f_{\mathrm{n}}$ can be seen embedded within the broadband hump. The dominant discrete frequency at which the largest sound pressure level occurs is referred to here as $f_{n-m a x}$.

After the different frequencies types $\left(f_{s}, f_{\mathrm{n}}\right.$ and $\left.f_{\mathrm{n} \text {-max }}\right)$ in a typical instability noise spectrum have been defined, Fig. 9a shows the measured noise spectral map versus flow velocity and frequency when the airfoil is set at $\theta=4.2^{\circ}$. The self-noise sound pressure levels are sufficiently large for 
several fine structures of "rungs" to be revealed in the colormap representation. Each rung represents a high amplitude tone mode that accurately follows the $v_{j}^{0.8}$ power-law whilst the general trend in the "averaged-tonal noise" follows the $v_{j}^{1.5}$ relationship. Note that the $v_{j}^{1.5}$ power-law for the averaged-tonal noise is in agreement with Paterson et al. [23]. The corresponding noise spectral map predicted using the empirical model developed by Brooks et al. [1] is shown in Fig. 9b. Figure 9c shows the total amplification level of the T-S wave, $A$, plotted as a function of flow velocity and frequency, calculated from Eq. (8), on the pressure surface of the airfoil. Comparisons with Brooks et al.'s results as well as the total amplification of the T-S waves are in very good agreement with the measurements. Figure $9 \mathrm{~d}$ provides a comparison of the frequencies of the dominant discrete tone at which the largest sound pressure level occurs $\left(f_{n-\max }\right.$, from the measured noise spectral), and the frequencies corresponding to the maximum T-S wave amplification factor $\left(f_{\text {TS-max }}\right.$, obtained from the linear instability calculation). The comparison provides a clear confirmation that the highest T-S wave amplification occurs at combinations of frequencies and flow velocities that closely match those of the measured and predicted highest sound pressure levels. The figures also reveal that both $f_{\mathrm{TS}-\max }$ and $f_{\mathrm{s}}$ (the averaged-tonal noise) $\alpha v_{\mathrm{j}}^{1.5}$, whereas both $f_{\mathrm{n}}$ and $f_{\mathrm{n}-\max } \alpha v_{\mathrm{j}}^{0.8}$.

Tam and Ju [8] have recently found that when $\theta=0^{\circ}$, all of the tone frequencies can fit well with the Paterson et al. [23] formula which is proportional to $v_{j}^{1.5}$. Because Tam and Ju only predict a single tone frequency at each velocity (or Reynolds number in their case), their findings imply that $f_{\mathrm{n}}$ $\max _{\alpha} \alpha v_{\mathrm{j}}^{1.5}$. Our experimental results for the similar airfoil at $\theta=0^{\circ}$ also agree with Tam and Ju's 1.5 velocity power law for the $f_{n-\max }$ (see Fig. $4 \mathrm{~d}$ of Ref. [3]). However, when $\theta \neq 0^{\circ}$, such as for $\theta=1.4^{\circ}$ (see Fig. $6 \mathrm{~d}$ of Ref. [3]) or $\theta=4.2^{\circ}$ (Fig. $9 \mathrm{~d}$ of the current paper), both $f_{\mathrm{n} \text {-max }}$ and $f_{\mathrm{n}}$ are instead found to be proportional to $v_{j}^{0.8}$. The transition of the velocity power law from a zero to a non-zero angle of attack implies that different noise mechanisms could be involved. This issue will be addressed further in Section 4.6.

\subsection{Identification of the separation region on the airfoil's surface with straight trailing edge, SO}

In order to establish the relationship between the location of the laminar separation and the corresponding instability tonal noise radiation, the separation region on the airfoil's pressure surface was investigated by the surface mounted hot-film arrays as described in Section 2.2.2. Figure 10 shows the simultaneously measured time-variation of the five hot-film signals, the phase angle, $\sigma$, between HF1-HF2, HF2-HF3 and so on, as well as their corresponding coherence functions, $\gamma^{2}$, for $v_{j}=$ $40 \mathrm{~ms}^{-1}$. The corresponding $f_{\mathrm{n}-\mathrm{max}}$ is indicated in the figures. Figure 10 shows that, even in a qualitative 
comparison of the simultaneously sampled raw time-history signals, there are many instances at which the phase shifts between HF1-HF2 and HF2-HF3 differ significantly, even though all sensors are separated by a same streamwise distance of $6 \mathrm{~mm}$. This phenomenon suggests that flow reversal might already occur in the neighbourhood of these sensors.

The visually determined discrepancy in the phase shift between the hot-film time signals can be further investigated from the phase spectral diagram. At the dominant tone noise frequency of $f_{n-m a x}$ where the coherences of the hot-film signals are reasonably high, the relative phase angle $\sigma$ for the $\mathrm{HF} 1-\mathrm{HF} 2$ case is $+137^{\circ}$ (from the absolute phase angle of $+857^{\circ}$ as determined from the diagram). This experimentally determined value can be verified by calculating the wavenumbers of the T-S instabilities $\alpha_{\mathrm{r}}$ from Eq. (3). The phase angle over a distance $\tau$ can be expressed as $\sigma=\alpha_{\mathrm{r}} \tau$. At the dominant frequency $f_{n-\max }$, the phase angle $\sigma$ for the HF1-HF2 case is calculated as $+156^{\circ}$, which agrees reasonably well with the experimental result. Note that a positive $\sigma$ implies that the predominantly sinusoidal instabilities are convected from HF1 to HF2 in the same direction as the main flow.

However, Fig. 10 also shows that the phase angles $\sigma$ for the HF2-HF3 case are negative for a similar frequency range. This implies that HF3 actually detects the coherent part of the signal ahead of HF2, which indicates that flow reversal at the near wall region has occurred at that location. Even though the coherence is low, the phase angles for the HF3-HF4 case also have a predominantly negative sign, which indicates that these sensors are also located inside the separation region. A physical explanation of the above results is proposed here: by referring to Fig. 11, a T-S wave is convecting from HF1 to HF2, where the onset of boundary layer separation is located near the location of HF2 (Top Picture). The T-S wave then enters the bubble, detaches from the surface and interacts strongly with the separating shear layer above the wall $[4,10]$ (Middle Picture). As it further propagates downstream, the near wall flow reversal of the bubble will transport some of the oscillatory disturbances originated from the T-S instability wave from the downstream hot-film sensors to the upstream hot-film sensors (Bottom Picture), thus resulting in a reverse phase shift as interpreted from the near wall hot-film array signals. In summary, the phase change of the convective T-S wave has been used for the determination of flow separation.

It should be mentioned that a reverse phase shift might not be detectable if the hot-film arrays are replaced with surface microphone arrays. This is because a microphone embedded underneath a test surface could also capture the disturbances of the propagating T-S and Kelvin-Helmholtz instabilities in the separating shear layer above the wall surface. 
The determination of the turbulent reattachment of the separation bubble is not satisfactory due to the low coherence obtained by the downstream hot-film sensors. Based on the results of the surface hot-film sensor arrays, the presence of boundary layer separation at around $x / C=0.83$ has been confirmed. It is important to point out that, in the current study, the roots of the serrated trailing edge $X_{\text {root }}$ for the S1, S2 and S3 cases are all located at $x / C=0.87$, which are fairly close to the point at which the boundary layer starts to separate. In Section 4.3 we will present results of an investigation to find out whether the trailing edge serration can trigger bypass transition and generate a turbulent-like boundary layer on its saw-tooth surface.

A last point to note is that an attempt was made to predict the separation point using the numerical code XFOIL which identifies the location of separation by the most upstream point at which the skin friction becomes negative. The separation points were predicted to occur at $x / C$ of about 0.83 across the jet velocity range [3], which is almost exactly the same as the current experimental value. This good degree of agreement suggests that the XFOIL is quite robust in predicting the boundary layer profiles developed on an airfoil even when the flow around the airfoil may be affected by a finite jet height.

\subsection{Growth of boundary layers on a saw-tooth trailing edge}

In view of the importance of the separated boundary layer to produce the multiple pure tones it is useful to discuss how a serrated trailing edge could possibly influence the behaviour of the separated boundary layer. As already established earlier, the global flow field around an airfoil will be unaffected irrespective of which type of trailing edge, straight or serrated, is used. Because of this, the sound pressure level and spectral characteristics of the radiated instability self-noise will respond to a serrated trailing edge through modifications of either the local acoustical scattering mechanism or the boundary layer. In this section, the growth of the boundary layer over a saw-tooth geometry is investigated and the implication of this on a separation boundary layer will be discussed.

Using hot-wire anemometry, boundary layer velocity profiles at several streamwise locations for $x / C=0.83,0.87,0.90,0.93$ and 0.97 were first measured along the pressure surface of an airfoil at $\theta$ $=5^{\circ}$ with a straight trailing edge (SO). The freestream velocity was set at $15 \mathrm{~ms}^{-1}$. After that the trailing edge was replaced with a serrated type (S3) and boundary layer measurements at the same streamwise locations, which are along the plane of symmetry of a saw-tooth, were repeated. Note that $X_{\text {root }}$ for S3 is located at $x / C=0.87$, thus the saw-tooth region is situated at $x / C>0.87$. Figures $12 \mathrm{a}-\mathrm{b}$ present the boundary layer mean $\left(u / U_{o}\right)$ and root-mean-square fluctuating velocity $\left(u_{\mathrm{rms}} / U_{o}\right)$ 
profiles, respectively, for the $\mathrm{S} 0$ and $\mathrm{S} 3$ cases. Note that $U_{0}$ is the local freestream velocity. In the region $x / C=0.90$ to 0.97 , the velocity profiles for the SO case are not stable and contain inflection points. ${ }^{*}$ However, when the trailing edge is replaced with the S3 type, the boundary layer velocity excess at the near wall region becomes more significant than in S0, resulting in a more stable boundary layer. The difference in the velocity profile shapes for these two types of trailing edges decreases as $x / C$ reduces from 0.97 to 0.90 . At $x / C=0.87$ ( $\left.X_{\text {root }}\right)$ and 0.83 , the velocity curves for the SO and S3 cases almost collapse, which indicate that the boundary layer upstream of the serration root is not affected by the saw-tooth. The readers are reminded that the results in Fig. 12 correspond to when the airfoil is set at $\theta=5^{\circ}$, which is different with the results discussed in Section 4.2 in which $\theta=4.2^{\circ}$. As shown in Fig. $12 \mathrm{~b}$, a similar development is also observed for the rms fluctuating velocity profiles. For streamwise locations $x / C \leq 0.87$, the rms profiles pertinent to the serrated S3 type are similar to the straight S0 type. However, for $x / C>0.87$ the near wall flow over a serrated trailing edge has a larger turbulence intensity level than the flow over a straight trailing edge. This important result indicates that the boundary layer on a serrated trailing edge is mostly turbulent in character and therefore is less likely to separate.

A further examination is carried out for the spectral content of the longitudinal velocity fluctuations for both the SO and S3 cases. To investigate the streamwise development of the boundary layer spectral content, at each frequency the velocity spectral $\Theta(\omega, y)$ were integrated across the boundary layer to obtain the overall boundary layer spectral energy level. The results are shown in Fig. 13 and as expected, the overall spectral energy levels for the SO case are low and their streamwise variations are also negligibly small. However, a peak at $538 \mathrm{~Hz}$ can be observed in all of the overall energy spectral diagrams in Fig. 13. This discrete component is unlikely to be associated with a separated boundary layer whose frequency is usually low and of broadband characteristic. This discrete component is most likely related to the propagating T-S waves frequency. To verify this, a linear stability analysis was undertaken of the boundary layer on the pressure surface of the airfoil developed under the same angle of attack and flow velocity. Figure 14a shows the corresponding contour map of the local T-S wave growth rate $\left(-\alpha_{i}\right)$. It can be seen that the first point where the instability starts to grow is situated at around $x / C=0.53$. The growth rate of the instability increases steadily in the streamwise direction. The maximum growth rate, however, varies slightly at each $x / C$ location but it generally centered at around $470-550 \mathrm{~Hz}$. The result presented in Fig. 14a can also be integrated to obtain the total amplification factor, $A$. As shown in Fig. 14b, the total T-S wave

\footnotetext{
* The separation point is also predicted by XFOIL to occur at $x / C=0.90$ in this case, which match well with the experimental results in Fig. 12.
} 
amplification factor peaks at $534 \mathrm{~Hz}$, which is very close to the dominant peak frequency $(538 \mathrm{~Hz})$ detected from the measured velocity energy spectral in Fig. 13 for the SO case.

The velocity energy spectral for the S3 case in Fig. 13, on the other hand, shows a different characteristic. A high level of spectral energy is evident in the saw-tooth region for $0.90<x / C<0.97$. The spectral energy was found to decay as $f^{-5}$ at the high frequency end without the presence of the $f^{-5 / 3}$ decay at the mid frequency region. The reason for the absence of this slower decay rate is possibly due to the turbulent boundary layer not fully developed and therefore remains inhomogeneous on the saw-tooth surface. The results in Fig. 13 are obtained by integrating the velocity spectral across the whole boundary layer at each frequency. In this case the lack of smaller turbulent eddies outside the buffer layer will cause a faster decay rate of the integrated spectral energy. This is analogous to the measurement of the unsteady wall pressure spectral from which a dominant spectral energy decay rate of $f^{-5}$ is reported for a turbulent boundary layer [24, 25]. Therefore, it can be confirmed that a serrated trailing edge will produce a bypass transition of the boundary layer, thereby influencing the radiated self-noise. There are two important points to note. First, the energy spectral at $x / C=0.83$ for the S3 case is associated with a laminar boundary layer slightly upstream of the saw-tooth surface. Second, the small peak at $538 \mathrm{~Hz}$, which was earlier proven in the straight S0 case to be associated with a T-S wave, is also visible for the serrated S3 case. These observations indicate that the radiation of the instability self-noise for a serrated trailing edge is still possible in regions close to the root of the saw-tooth $X_{\text {root }}($ at $x / C=0.87$ ) although at the same time the spectral level and tone frequency may be different compared to the straight trailing edge counterpart (this issue will be addressed in Section 4.5).

As a summary of this section, the increased turbulence characteristics and the tendency of the boundary layer to remain attached on the pressure surface in the presence of a serrated trailing edge have important implications on the behaviour of the separated boundary layer and the noise scattering location ( $\varphi_{2}$ in Eq. 8). These combinations will eventually lead to modifications of the total amplification level of the T-S wave, $A$, the level of the radiated broadband hump and the frequencies of the multiple pure tones. This important causal link will be reflected in Section 4.4 and further discussed in Section 4.5.

\subsection{Comparisons of airfoil instability noise with straight and serrated trailing edges}

Figure 15 shows comparisons of the instability noise spectra with and without trailing edge serrations at $\theta=4.2^{\circ}$ for different combinations of $\left(\varepsilon, X_{\text {root }}\right)$. As discussed earlier, the separation point 
is located at approximately $x / C=0.83$. For serrations with $X_{\text {root }}=0.87$ (S1, S2 and S3), the separation point on the pressure surface is located slightly upstream of $X_{\text {root }}$. Based on the results presented in Sections 4.2 and 4.3, the serrated trailing edges S1, S2 and S3 can inhibit a very significant part of the separation region. The effects of an absence of a separation region on the radiated noise can be clearly seen in Fig. 15a, b and d, where neither the broadband hump nor the multiple discrete tones are present. Therefore some large tonal noise reductions have been achieved by the S1, S2 and S3 serrated trailing edges. However, for a serrated trailing edge with a more downstream $X_{\text {root }}$ at 0.93 $\left(\mathrm{S2}{ }^{\prime \prime}\right)$, the broadband hump and some discrete tones remain (see Fig. 15c). This is because the $X_{\text {root }}$ in this case is located further downstream, thus allowing some degrees of amplification due to the T-S wave/separating shear layer interaction from $x / C=0.83$ to 0.93 , before being scattered into tonal noise near the root of the serrated edges.

The Overall Sound Pressure Level (OASPL) of the airfoil noise at a particular flow velocity can be expressed as:

$$
\text { OASPL }=10 \log _{10}\left(\frac{\int \bar{P}^{2}(f) d f}{4 \times 10^{-10}}\right)
$$

In this study the OASPL was obtained by integrating the mean square pressure ${\overline{P^{\prime}}}^{2}$ over the frequency range, $0.1 \mathrm{kHz}-15 \mathrm{KHz}$, where the lower bound limit represents approximately the cut-off frequency of the anechoic chamber. Fig. 16a shows the variation of OASPL with mean velocity $v_{\mathrm{j}}$ for the straight and various serrated trailing edges. The background noise of the open jet wind tunnel is also included in the figure which has been shown to be at least $30 \mathrm{~dB}$ and $10 \mathrm{~dB}$ lower than the self noises produced by the straight and serrated trailing edges respectively. Initially the OASPL for SO, S1, S2 and S3, as well as S2", remain nearly constant at $20 \mathrm{~ms}^{-1}<v_{\mathrm{j}}<24 \mathrm{~ms}^{-1}$ where the instability tonal noise is absent. For the SO case, the tonal noise begins to occur at $24 \mathrm{~ms}^{-1}<v_{\mathrm{j}}<30 \mathrm{~ms}^{-1}$. From $v_{\mathrm{j}}$ $=30 \mathrm{~ms}^{-1}$ to $60 \mathrm{~ms}^{-1}$, the instability noise radiation continues to be dominant with a speed dependence of $\mathrm{OASPL}_{\left(S_{0}\right)} \propto v_{\mathrm{j}}^{5}$ corresponding to the classical trailing edge noise radiation. In this velocity range the spectrum is characterized by numerous and equally-spaced discrete frequencies (sometimes as many as 10 at a single velocity). Identical behaviour is found in the experimental and numerical works of $[2,3,5,7,23]$. For the $\mathrm{S} 1, \mathrm{~S} 2$ and $\mathrm{S} 3$ serrations, there is no sudden jump in OASPL at $20 \mathrm{~ms}^{-1}<v_{\mathrm{j}}<60 \mathrm{~ms}^{-1}$ and it is found that $\mathrm{OASPL}_{(\mathrm{s1}, \mathrm{s2}, \text { and } \mathrm{s3})} \propto v_{\mathrm{j}}^{7}$. The increase in the velocity power law from 5 to 7 demonstrates that noise radiation at the trailing edge has largely been inhibited and the dominant noise sources are now mainly arising from the quadrupole sources in the jet and the airfoil turbulent wake. For the S2" serration, although the OASPL levels still scale roughly with $v_{\mathrm{j}}{ }^{7}$, 
they are generally higher level than in the other serration cases (S1, S2 and S3) at $v_{\mathrm{j}}>30 \mathrm{~ms}^{-1}$. This is because some lower level instability tonal noises have been known to exist for this type of serration (Fig. 15c).

Figure $16 \mathrm{~b}$ shows the difference in the Overall Sound Pressure Level $\triangle O A S P L$ between a baseline straight trailing edge (S0) and a serrated trailing edge (S1, S2, S3, or S2"). Appreciable noise reductions of up to $20 \mathrm{~dB}$ occur at $v_{\mathrm{j}}>24 \mathrm{~ms}^{-1}$. By comparing serrations $\mathrm{S} 1, \mathrm{~S} 2$ and S3 of the same $2 h$ (serration length) but different $\varepsilon$ (serration angle) the level of noise reduction increases as $\varepsilon$ increases. For the pair of serrations S2 and S2" with the same $\varepsilon$ but different $2 h$, an increased level of noise reduction is achieved by the serrated trailing edge with a larger $2 h$. This is because a larger part of the separation region has been inhibited, which then weakens the amplification process of the T-S waves and the efficiency of tonal noise radiation.

Supplementary noise measurements were performed in order to further examine the velocity dependency of the self noise produced by a serrated trailing edge. A detailed description of the experimental set up can be found in Chong et al. [26]. Figure 17 shows the variations of the Overall Sound Power Level (OAPWL) with mean velocity for the straight SO and serrated S3 trailing edges in two cases. In the first case the boundary layer tripping elements are applied near the leading edges of the suction and pressure surfaces (Case A), which would eventually produce turbulent broadband noise. In the second case no boundary layer tripping elements are applied (Case B), which would produce predominantly laminar instability tonal noise. From the figure, it is clear that in Case A $\mathrm{OAPWL}_{(50 \text { and S3) }} \propto v_{\mathrm{j}}^{5.5-6}$. This result indicates that the broadband noise radiation can largely be scaled with a non-compact dipole, which confirms the dominance of the edge-scattered noise source in Case A regardless whether the trailing edge is of straight or serrated type. On the other hand, in Case B it can be found that $\mathrm{OAPWL}_{(\mathrm{SO})} \propto v_{\mathrm{j}}^{5}$ and $\mathrm{OAPWL}_{(\mathrm{S} 3)} \propto v_{\mathrm{j}}^{7}$. The velocity dependencies are similar to the OASPL results presented earlier. By referring to the discussions in Section 4.3 , the $v_{j}^{7}$ dependency for a serrated trailing edge in Case B could be explained by the fact that the newly formed turbulent boundary layer on the saw-tooth surface is inhomogeneous and could not afford an effective conversion of the hydrodynamic energy into the acoustic energy from the saw-tooth side edges and tips. This can be supported by the fact that OAPWL produced by the S3 serrated trailing edge in Case B is lower than the counterpart in Case A. As a result, the dominant noise source produced by an airfoil with either a S1, S2 or S3 serrated trailing edge in Case B is not likely to originate at the trailing edge, although a lower degree of noise scattering at $X_{\text {root }}$ for the S2" serrated case is known to exist. 


\subsection{Effects of trailing edge serration on the aeroacoustic feedback models}

\subsubsection{Noise radiation}

After the integrated noise levels in the form of OASPL have been discussed in Section 4.4, it is of interest to investigate the instability noise spectral characteristics in greater detail. In particular how the multiple discrete tonal frequencies vary with the jet velocity when trailing edge serrations are introduced. First of all, Fig. 18 shows the contour of sound pressure level versus frequency and jet velocity for a straight trailing edge (SO) at $\theta=4.2^{\circ}$. From the figure, several high intensity acoustic "rungs" with a power-law of about 0.8 (i.e. $f_{\mathrm{n}} \alpha v_{\mathrm{j}}^{0.8}$ ) can be seen. The values of $f_{\mathrm{n} \text {-max }}$ (frequency that corresponds to the most dominant tone at a particular velocity) at a range of jet velocities were also superimposed in the figure. It can be observed that over a small velocity range, the distribution of $f_{\mathrm{n}}$

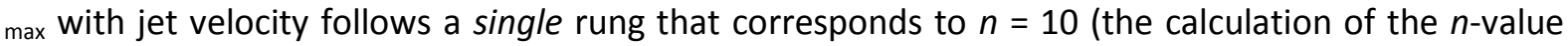
will be explained in Section 4.5.2) and also follows the 0.8 power law. As the velocity increases, the frequency jumps to another parallel rung $(n=12)$. When the velocity is increased further and approaching $60 \mathrm{~ms}^{-1}, f_{\mathrm{n}-\max }$ jumps to yet another rung $(n=16)$. The above phenomenon, which is usually referred to as the "ladder structure" and its formation is exclusively related to $f_{n \text {-max }}$ is well documented for straight trailing edge airfoils $[5,23,27]$.

The corresponding acoustical contour maps for the cases when the trailing edge is replaced by serrations of type S2 and S2" are shown in Figs. 19a and 19b respectively. Note that both S2 and S2" serrations have the same serration angle, $\varepsilon=12^{\circ}$, but with $X_{\text {root }}$ at 0.87 and 0.93 respectively. No rungs, i.e. tones, are discernible in the S2 case, as shown in Fig. 19a. This is because amplification of the T-S wave has been weakened due to the tendency of this particular type of serration to suppress separation where the separation point has been shown to occur at $x / C=0.83$ whereby $X_{\text {root }}=0.87$. However, for the S2" serration, where $X_{\text {root }}=0.93$, regions of separated flow upstream of the root of the saw-tooth still exist, which allow a considerable amplification of the T-S wave to take place at $0.83<x / C<0.93$. As a consequence, some acoustic rungs remain in Fig. $19 \mathrm{~b}$ as the results of tonal noise radiation at $X_{\text {root }}=0.93$, although in general they are fewer than in the S0 case. Moreover, the noise levels of the rungs appear to be lower, which suggest that the intensity of the radiated instability noise is dependent upon the length of the separation region and therefore on the amplification levels of the T-S waves by the separated flow.

As shown in Fig. 19b, one important point to note is that although the variation of $f_{\mathrm{n} \text {-max }}$ with jet velocity for the S2" serration was found to follow the 0.8 power law, it remains on the same rung (at $n=11$ ) over the entire range of velocities under investigation here. Unlike the case of a straight 
trailing edge, where the variation of $f_{n-\max }$ with velocity has been found to undergo several abrupt "jumps", the ladder structure ceases to exist when a S2" serration is introduced at the trailing edge. The mechanism that causes this delay/disappearance of the ladder structure will be discussed in Section 4.5.3.

Figures 20a-c represent the noise contour maps, as a function of frequency and jet velocity, pertaining to the S1, S2 and S3 serrations respectively. These serrations share the same $X_{\text {root }}$ but with different serration angles $\varepsilon$. No acoustic rung is discernible for the S2 and S3 serrations and a complete suppression of the tonal noise is apparent. The S1 serration, which has the shallowest serration angle $\varepsilon$, only exhibits a single acoustic rung at $v_{\mathrm{j}}>50 \mathrm{~ms}^{-1}$. Therefore, no ladder structure is found for this case. We can therefore interpret from Fig. 20a-c that a wider serration angle $\varepsilon$ is more effective in preventing the formation of a separated boundary layer, which ultimately reduces the tendency of instability tonal noise radiation. This is in line with the OASPL distributions as presented in Fig. 16.

\subsubsection{Assessment of feedback mechanism based on Model A}

The mechanism for the generation of multiple discrete tones can be related to one, or a combination, of the aeroacoustic feedback models described earlier (see Fig. 1). Based solely on Model $\mathrm{A}$, the tonal frequencies can be predicted $[2,3,5]$ from the frequencies, $f_{n}$, at which the phase change around the loop $2 \pi F\left(f_{n}\right)$, given by

$$
F\left(f_{\mathrm{n}}\right)=\frac{C}{2 \pi} \int_{\varphi_{1}}^{\varphi_{2}} \alpha_{\mathrm{r}}\left(\frac{x}{C}\right) d\left(\frac{x}{C}\right)+\frac{f_{\mathrm{n}} L}{c_{0}-U_{\infty, L}}+\frac{1}{2}
$$

are integer multiples of $2 \pi$, i.e.

$$
F\left(f_{\mathrm{n}}\right)=n \quad(n=1,2,3 \ldots)
$$

where $\alpha_{\mathrm{r}}$ is the wavenumber of the T-S wave convecting from $\varphi_{1}$ (the first point of instability) to $\varphi_{2}$ ( 0.99 for straight trailing edge or $X_{\text {root }}$ for serrated trailing edge). The complex wavenumbers were calculated from solutions of the Orr-Sommerfeld equation governing the eigenvalues of 2D sheared viscous flows using the linear stability analysis described in Section 3. The second term on the right hand side of Eq. (11) accounts for the phase change due to the acoustic wave propagating from $\varphi_{2}$ back to $\varphi_{1}$. Note that $c_{o}$ is the speed of sound, $L=\left[\left(\varphi_{2}-\varphi_{1}\right) \times C\right]$ and $U_{\infty, L} L$ is the average freestream 
velocity between $\varphi_{1}$ and $\varphi_{2}$. The factor of $1 / 2$ in Eq. (11) accounts for the $180^{\circ}$ phase change due to the Kutta condition at the trailing edge $[2,3,5,28]$.

The numerous discrete tones present in the measured noise spectra, such as those in Figs. 15, 18 and 19 , indicate that at a given velocity, $F\left(f_{n}\right)$ is satisfied for a wide range of $n$ values. Each discrete frequency $f_{n}$ thus corresponds to solution where the phase function $F\left(f_{n}\right)$ takes an integer value $n$. Applying this argument to the SO straight edge at $\theta=4.2^{\circ}$, the discrete frequencies $f_{\mathrm{n}}$ satisfying Eq. (11) over a range of velocities were calculated. The variations of $f_{\mathrm{n}}$ with $v_{\mathrm{j}}$ computed from Eq. (11) are superimposed on Fig. 18. Note that the theoretical discrete frequencies $f_{n}$ also follow a velocity power law of 0.8 for the even values of $n: 10,12,14$, and 16 which match the measured tonal rungs very well.

An identical analysis was performed for the serrated trailing edge airfoils. Predictions of the discrete frequencies, according to Eq. (11), for the saw-tooth geometry were computed from the phase change between $\varphi_{1}$ and the root of the saw-tooth of serration $S 2^{\prime \prime}, X_{\text {root }}=0.93$ where most of instability scattering begins to occur. The theoretical discrete frequencies are superimposed on the noise contours of Fig. 19b. The relationship $f_{\mathrm{n}} \alpha v_{\mathrm{j}}^{0.8}$ is also observed when serration is introduced. Furthermore, the measured rungs are shown to match well with the predicted $f_{\mathrm{n}}$ values for odd integer $n$-values: $n=11,13$ and 15, compared with the even $n$-values for the straight edge airfoil S0. The disparity of the $n$-values obtained for the S0 and S2" airfoils, respectively, can be attributed to the modification of the aeroacoustic feedback loop structure. When the acoustic scattering location is shifted upstream by the S2" serration (i.e. $\varphi_{2}$ is reduced), the $L$ term in Eq. (11) is also reduced. Because the trailing edge serration will not change the global flow field around the airfoil significantly, we can reasonably assume that $\varphi_{1}$ remains unchanged. Therefore the first to terms on the right hand side of Eq. (11) will be affected when a serrated trailing edge is used. In order to achieve the nearest integer value of the phase function $F\left(f_{n}\right)$ for the $S 2$ " case, the tone frequencies $f_{n}$ need to be slightly shifted. Hence, the tonal rung that matches $n=12$ for the so case roughly corresponds to the tonal rung that matches $n=11$ for the S2" case, and so on.

\subsubsection{On the variations of $f_{n-\max }$ with velocity for a straight trailing edge, $\mathrm{S} 0$ and a serrated}

\section{trailing edge, S2"}

In a previous investigation of the variations of $f_{n-\max }$ with velocity at a smaller angle of attack at $\theta$ $=1.4^{\circ}$ for the SO case [27], we have experimentally verified that the ladder structure is a result of the acoustic/hydrodynamic frequency lag between the scattering of the T-S wave instability noise $\left(f_{s}\right)$ at 
the trailing edge and the discrete noise $\left(f_{n}\right)$ produced by an aeroacoustic feedback loop based on Model A. A schematic to summarize this mechanism is presented in Fig. 21. At a small angle of attack, symmetric can be seen about the maximum peak in the broadband hump at $f_{s}$, where the pairs of the discrete tones $\left(f_{1}, f_{5}\right)$ and $\left(f_{2}, f_{4}\right)$ are equidistant to the line of symmetry as depicted in (I). During this stage $f_{3}=f_{n-\max }=f_{\mathrm{s}}$. As illustrated in (II), when the flow velocity increases, $f_{\mathrm{s}}$ increases at a faster rate $\left(\sim v_{\mathrm{j}}^{1.5}\right)$ than any of the discrete tones $f_{\mathrm{n}}\left(\sim v_{\mathrm{j}}^{0.8}\right)$ and therefore all of the $f_{n}$ start to lag with respect to the $f_{\text {s. }}$. At some points in (III), a new tonal mode will eventually replace the previous most dominant tone to coincide with the peak of the broadband hump (e.g. $f_{4}$ instead of $f_{3}$ has now become the $\left.f_{n-m a x}\right)$. These jumps in the most dominant frequency give rise to the aforementioned ladder structure.

Therefore an important criterion for initiating and sustaining a ladder structure is the existence of a broadband hump because the hump peak essentially acts as a "carrier" for the most dominant tone frequencies $f_{\mathrm{n} \text {-max }}$. Compared with the $\mathrm{S} 2$ " serration case where $\varphi_{2}=0.93$, the total amplification level $A$ (defined in Eq. 8) for the T-S waves in the straight SO case will be larger because $\varphi_{2}(=0.99)$ is larger. This effect is clearly manifested in the radiated noise spectra in Fig. 15c where the broadband humps are less significant for the $\mathrm{S} 2$ " serration when compared with the SO straight trailing edge. Another effect of the serration S2" on the ladder structure is that the $\Delta f$, which is defined as $f_{n+1}-f_{n}$, is larger than on the So case. This means that the ladder-jumping, from one acoustic rung to another, should now occur over a larger margin of velocity increase.

The introduction of serrations, as discussed above, is responsible for producing the spectral characteristics in Fig. 19b, whereby the frequency bandwidth for each of the acoustic rungs is now narrower compared to the straight edge S0 case in Fig. 18. This essentially means that the broadband hump feature is also weakening. Consequently, no ladder jump has occurred for $v_{j} \leq 60$ $\mathrm{ms}^{-1}$ in the present study for the $\mathrm{S} 2$ " serration case. Nevertheless, it is still possible that a ladder jump might eventually occur for $v_{j}>60 \mathrm{~ms}^{-1}$. This is because the sound pressure levels for the second rung ( $n=13)$ and the third rung $(n=15)$ have been observed to start becoming more significant when $v_{\mathrm{j}}$ gets close to $60 \mathrm{~ms}^{-1}$ and may eventually overtake the sound pressure level for the primary rung at $n=11$. However, this remains speculative at the moment because no noise data is available for $v_{\mathrm{j}}>60 \mathrm{~ms}^{-1}$. 

acoustical properties

The aeroacoustic feedback Model A, which does not take the airfoil wake flow into consideration, has been found to be reasonably accurate in the prediction of the discrete tone frequencies $f_{\mathrm{n}}$ when the NACA-0012 airfoil is set at $\theta=4.2^{\circ}$. It was hypothesized that at a fixed Reynolds number and particularly at a very low angle of attack the generation of the discrete tones could involve more than one aeroacoustic feedback mechanism [6, 7]. Based on the feedback Model A, Equations (11) and (12) assume that the loop occurs between the point on the airfoil pressure surface where instabilities start to develop and the trailing edge (or $X_{\text {root }}$ for a serrated trailing edge). However, in the DNS study by Desquesnes et al. [7], a noise source in the wake was taken into consideration in an attempt to explain the mechanism of tone noise generation. They proposed an aeroacoustic feedback loop between the point at which instabilities start to develop on the airfoil's surface and a dipole source downstream of the trailing edge in the wake (Model B in Fig. 1). Tam [6] has proposed a third loop structure between the trailing edge and a dipole source in the wake (Model C in Fig. 1). More recently, Tam and Ju [8] propose that when $\theta=0^{\circ}$ the tone is produced by the interaction between the trailing edge and the near wake Kelvin-Helmholtz instability, although the role of the feedback Model C in their case is not clear as only a single tone frequency is predicted at each Reynolds number.

The "dipole" noise sources proposed by Desquenes et al. and Tam as part of feedback Models B and $\mathrm{C}$ are consistent with the region within the near wake where an absolute instability is found to dominate. For example, in an experimental study of the flow behind flat plates and cylinders, Mattingly and Criminale [29] observed a localized velocity oscillation, which can only be sustained in the absolute instability region. Koch [30] proposed a global resonance condition in which wake tones can sometimes be emitted. For the case of an airfoil in low Reynolds number flows, the near wake could be characterized by an absolute instability provided that the local adverse pressure gradient level is sufficiently large [31, 32].

In the present work, PIV measurements were conducted separately in a closed-section wind tunnel to measure the airfoil wakes for both the straight (SO) and serrated (S2) trailing edge cases in order to verify the existence of wake-based sources. In order to investigate the role of the dipole noise source in the tonal noise generation mechanism, PIV measurements were focused on the wake flow regions. Figures $22 \mathrm{a}$ and $22 \mathrm{~b}$ show the contour maps of the rms of the normal-component velocity fluctuations, $v_{\text {rms }}$ of the straight trailing edge case, $\mathrm{S} 0$ at two angles of attack, $\theta=0^{\circ}$ and $2^{\circ}$ 
respectively ${ }^{\dagger}$. In both cases the inlet freestream velocities were set at $15.9 \mathrm{~ms}^{-1}$. In Fig. 22a, a localized velocity fluctuation can be seen at approximately $x / C=0.07$ from the trailing edge. This localized region resembles closely the dipole noise source suggested in a number of other papers [6, 7]. At $\theta=2^{\circ}$, the localized fluctuation/dipole noise source is still present, as shown in Fig. 22b. However, the localized region is now situated closer to the trailing edge at approximately $x / C=0.02$ from the trailing edge.

The above measurements were repeated at the same flow conditions using the S2 serrated trailing edge. The resulting $v_{\text {rms }}$ contour maps are plotted in Figs. 23a and 23b for $\theta=0^{\circ}$ and $2^{\circ}$ respectively. Comparison of the figures confirms that the wakes generated by the serrated trailing edge are more turbulent than those generated from the straight edge airfoil. This is consistent with the results presented in Section 4.3 in that a saw-tooth geometry can produce bypass transition of the boundary layer into a turbulent state. Subsequently, turbulent eddies shed into the wake flow region would affect the instability mechanism as well as the noise radiation mechanism.

We now investigate the relationship between the wake flows (Figs. 22 and 23) and their radiated noise characteristics. Whilst the wind tunnel was acoustically noisy, the principal airfoil tones clearly protrude above the background noise levels by at least $5 \mathrm{~dB}$ and in some cases, by $35 \mathrm{~dB}$. Figure 24 compares the spectra of the airfoil noise at $\theta=0^{\circ}$ for the S0 and S2 airfoils. Note that the presence of tones for the S2 serrated trailing edge at zero degree angle of attack is because the laminar separation bubble occurs at a more upstream location from the trailing edge due to the stronger adverse pressure gradient level. The amplitudes of the dominant tones for the two cases clearly differ in their intensities and frequencies. To investigate the reasons behind these discrepancies, feedback Model A is again used to calculate the theoretical tone frequencies pertinent to the two cases. The predicted and measured tone frequencies from Fig. 24 are summarized in Table 1. Consider the SO straight trailing edge, both of the predicted and measured tone frequencies are in excellent agreement for $n=9$ (about $540 \mathrm{~Hz}$ ) and $n=10$ (about $606 \mathrm{~Hz}$ ). However, the most dominant tonal frequency, $f_{\mathrm{n} \text {-max }}$ from the measurement occurs at $572 \mathrm{~Hz}$, which lies between the above $n$-values. For the S2 airfoil serration, the measured value of $f_{n-\max }$ occurs at about $540 \mathrm{~Hz}$, which corresponds well with the predicted frequency of $541 \mathrm{~Hz}$ at $n=8$. The results of Table $1 \mathrm{imply}$ that, for the SO straight trailing edge, the non-wake based feedback Model A cannot predict the dominant tone at $572 \mathrm{~Hz}$. By contrast, this tone is likely to be produced by a wake-based source that might involve a feedback mechanism such as in Models B or C. On the other hand, the dominant

\footnotetext{
${ }^{\dagger}$ Note that the geometrical angle of attack $\theta_{\mathrm{g}}$ is found to be close to the effective angle of attack $\theta$ in a closed working section wind tunnel. As a result the notation of $\theta$ is also adopted here to represent the angle of attack.
} 
tone for the S2 airfoil serration is consistent with feedback Model A. As shown in Fig. 23a, a turbulent wake is formed downstream of the serrated trailing edge, which inhibits the generation of wake tones.

The analysis of the relationship between the wake flows and the tone frequencies continues here for the $\theta=2^{\circ}$ case. Figure 25 compares the spectra of the airfoil noise for the straight SO and serrated S2 trailing edges, and Table 2 compares the predicted and measured tone frequencies. The good level of agreement in this case suggests that at this angle of attack, feedback Model A can adequately predict all the measurable tones from the experiment, for both the S0 and S2 trailing edges. Consider again the SO straight edge case, excellent agreement is achieved between the predicted and measured tonal frequencies for $n=8$ (about $517 \mathrm{~Hz}$ ) and $n=9$ (about $575 \mathrm{~Hz}$, dominant tone). Since the localized normal-component velocity fluctuations are also observed in the aerodynamic measurement (Fig. 22b), a wake-based tone could still be generated in this case. At $\theta=$ $2^{\circ}$, we observed that both Models $A$ and $B$ for the SO straight edge case contain similar feedback structure $^{\ddagger}$ because the localized normal-component velocity fluctuation is very close to the trailing edge (Fig. 22b). Therefore both models are likely to predict similar discrete tone frequencies, which explain that feedback Model $A$ is able to predict with reasonable accuracy the principal tone frequencies of a SO straight edge airfoil at $\theta=2^{\circ}$. This point can further be supported by the observation that when a turbulent wake is formed due to the S2 serrated trailing edge (Fig. 23b), feedback Model A (based on $\varphi_{2}=0.87$ ) can also adequately predict the radiated tone frequencies.

\section{Conclusions}

The results of an experimental study have been described to establish the effect of trailing edge serrations on airfoil self-noise at low to medium Reynolds numbers. Aeroacoustic measurements were performed in a quiet, low turbulence open jet wind tunnel located within a large anechoic chamber. The test was performed at a moderate angle of attack at $\theta=4.2^{\circ}$, covering Reynolds numbers between $1 \times 10^{5}$ and $6 \times 10^{5}$.

The ability of a trailing edge serration to reduce the airfoil tonal noise is confirmed in this paper. In an effort to investigate the two main serration parameters, namely the serration angle $\varepsilon$ and the serration length $2 h$, it has been generally found that a large reduction of the tonal noise level can be

\footnotetext{
* The feedback loop structure about the wake noise source and the suction surface of the airfoil in Model B is ignored in the present case. This assumption should be reasonably valid given that, in our previous study [3], we cannot identify any significant role of the boundary layer flow at the suction surface in contributing the instability tonal noise radiation across a wide range of Reynolds numbers.
} 
achieved when both $\varepsilon$ and $2 h$ are sufficiently large. The underpinning mechanisms that cause this phenomenon could be related to two main elements that have already been identified by others: the Tollmien-Schlichting (T-S) waves and the separation bubble. These two elements are important for an effective tonal noise radiation of an airfoil.

The spectral characteristics of the T-S instability wave and the radiated acoustic wave from the airfoil's trailing edge are found to be strongly correlated to each other. Therefore, the hydrodynamic instability indeed represents an important tonal noise source. An assumption was made that a separation bubble is also essential for the radiation of the airfoil instability tonal noise because it is needed to amplify the incoming T-S waves $[4,10]$. In this paper, a technique was developed to identify whether a separation region is present at the pressure surface of the NACA-0012 airfoil. Because of the near wall reverse flow characteristic for a separated flow, the phase shift of the convective T-S wave in the longitudinal direction has been successfully used to identify the separation point, which is found to occur close to $X_{\text {root }}$ of the S1, S2 and S3 serrated trailing edges. This has a significant impact on the state of the boundary layer at $x / C>X_{\text {root }}$ for a serrated trailing edge. Experimental evidences provided in this study confirmed that bypass transition will occur at $x / C>X_{\text {root }}$ where the boundary layer will become turbulent-like; on the other hand, for $x / C \leq X_{\text {root, }}$ the boundary layers will have the same characteristics as for the straight edge counterpart. Separation occurring in the straight trailing edge case is expected to be inhibited at $x / C>X_{\text {root }}$ for a serrated trailing edge. Therefore a saw-tooth geometry not only reduces the two-dimensionality of the T-S wave near the trailing edge, but also inhibits the amplification process that is supposed to take place at $x / C>X_{\text {root}}$. Therefore, as long as the separation region is located near the trailing edge of an airfoil, the combination of the above effects ultimately causes the reduction of the instability tonal noise level.

Whilst the noise control aspect has been addressed in a relatively straightforward manner, a significant part of this paper is devoted to investigate the airfoil instability noise mechanisms through the use of trailing edge serrations. Special emphasis was placed on the aeroacoustic feedback loop concepts. Three general models have been proposed in the literature - Models A, B and $\mathrm{C}$ as depicted in Fig. 1. In contrast to Model A, both Models B and C involve a prominent noise source in the wake. Sound pressure level contours versus frequency and velocity were presented. For straight SO and serrated S2" trailing edges, several high intensity tonal rungs were clearly observed. Predictions of these discrete tonal frequencies, based on the feedback Model $A$, are shown to be in close agreement with the experimentally observed frequencies for the SO case. Good agreement is also obtained for the serrated trailing edge S2" case. The serrated trailing edge has 
been shown to shift the scattering location, $\varphi_{2}$, to a more upstream location, thereby shortening the length of the feedback loop $L$ in Model $A$ and the overall phase change of the complex wavenumbers, which eventually causing a global shift of the discrete tone frequencies and the acoustic rungs (compare Fig. 18 and Fig. 19b).

More detailed aerodynamic measurements were performed using the PIV technique. Special emphasis was placed on the airfoil wakes pertaining to both straight SO and serrated S2 trailing edges. For the SO case, it was found that localized, non-convective normal-component velocity fluctuations are prominent inside the wake. This aerodynamic feature, which has been both predicted and measured previously $[6,7,29]$, can be considered as a noise source. The distance of this localized velocity fluctuation/noise source from the trailing edge is shown to vary with the airfoil's angle of attack. In the case of a large longitudinal separation between the trailing edge and the localized velocity fluctuation inside the wake (when $\theta=0^{\circ}$ ), a tone frequency additional to those predicted by feedback Model A is observed experimentally. This additional tone can be attributed to the existence of a wake-based feedback mechanism such as in the feedback Models B or C, or it may also be produced without any feedback mechanism at all. When a serrated S2 trailing edge is introduced at the same angle of attack, a turbulent wake is formed that suppresses the growth of the wake instability. In this case there will be no contribution from the wake flow and all radiated tones for the $\mathrm{S} 2$ case are attributed solely to the feedback Model A.

When the longitudinal separation between the trailing edge and the wake-based noise source is small (when $\theta=2^{\circ}$ ), all of the measured discrete tone frequencies can be predicted reasonably well by feedback Model A. This is because in this case the feedback Models A (a non-wake model) and B (a wake-based model) are now similar in structure. This point can be confirmed by the fact that when a serrated S2 trailing edge is introduced at the same angle of attack, the $f_{n \text {-max }}$ occurs at a frequency which is quite close to the $f_{n-\max }$ of the SO case (Table 2) even though the structures of the feedback Model A can be slightly different between the SO and S2 cases due to the different $\varphi_{2}$ and L.

At an even larger angle of attack at $\theta=4.2^{\circ}$, where more detailed acoustic results are available, all of the $f_{\mathrm{n}}$ and $f_{\mathrm{n} \text {-max }}$ can be predicted accurately by the non-wake based feedback Model $\mathrm{A}$ regardless whether the trailing edge is of straight S0 or serrated S2" type. This indicates that no wake-based noise source is present at this angle of attack, which can be further confirmed by our additional PIV results on the airfoil wake flow at $\theta=5^{\circ}$ (see Fig. $6 \mathrm{c}$ in Chong and Joseph [33]). 
To summarize the above findings, as far as the NACA-0012 airfoil is concerned, the laminar instability noise mechanism is more complex for $\theta<1^{\circ}$ where at least two tone noise sources or mechanisms may exist simultaneously, especially at low Reynolds number flow.

Another airfoil instability noise mechanism, namely the "ladder" structure, has also been studied in the current paper. The ladder structure has been proposed to be caused by the acoustic/hydrodynamic frequency lag between the scattering of the T-S wave in the form of broadband hump, with its peak frequency at $f_{\mathrm{s}}$, and the multi-discrete tone noise $f_{\mathrm{n}}$ produced by an aeroacoustic feedback loop [27]. Using the straight SO trailing edge several abrupt jumps in $f_{n-m a x}$ with changes in flow velocity can be observed (Fig. 18). When the trailing edge is replaced by the serration type S2", however, the $f_{n-m a x}$ do not exhibit any sudden jump in frequency across the entire range of flow velocities under investigation in the current study (Fig. 19b). Without firmly asserting that a serrated trailing edge can completely suppress a ladder structure, the non-ladder jumping phenomenon for the $\mathrm{S2}$ " case could be caused by the combined effects of:

(1) Larger $\Delta f$, which is defined as $\left(f_{n+1}-f_{n}\right)$. In other words, a larger margin of velocity increase is required in order to "shift" the $f_{\mathrm{n}}$ and $f_{\mathrm{n}+1}$ across $f_{\mathrm{s}}$ sequentially, which is the condition for a ladder jump to occur.

(2) Lower amplification factor $A$ for the T-S waves, which can result in a radiation of lower noise levels for the broadband hump peak at $f_{s}$. This phenomenon will proportionally reduce the noise level difference for $f_{\mathrm{n}}$ and $f_{\mathrm{n}+1}$, thus making an identification of a ladder jump event more difficult.

Finally, we believe that the tone noise generated in this experimental study is of genuine tones of an isolated airfoil. This can be supported by the fact that, when considering either a straight trailing edge or a serrated trailing edge, the overall airfoil geometry at the same angle of attack is still retained and the wind tunnel setup and locations of the laboratory equipment, which could potentially become an anchor point for the acoustic feedback loop, are exactly the same. However, the straight S0 and serrated S2" trailing edges have been shown to produce systematically different spectral characteristics, especially in the forms of tonal rungs, which can be predicted accurately by the original and slightly modified acoustic feedback Model respectively. In summary, the trailing edge serration is a useful device for the suppression of airfoil instability self-noise. For greater control effectiveness, however, the laminar separation bubble must be situated within the serration region of the trailing edge. This connection imposes restrictions on the angle of attack and velocity over which trailing edge serrations are effective. 


\section{Acknowledgments}

This work is partly supported by the BRIEF award from Brunel University. We would like to thank Dr Michael Kingan for allowing us to use his code for solving the Orr-Sommerfeld equation, and the anonymous referees for their suggestions to improve the paper.

\section{References}

[1] T. F. Brooks, D. S .Pope, M. A. Marcolini, Airfoil self-noise and prediction, NASA Reference Publication No. 1218, 1989.

[2] H. Arbey, J. Bataille, Noise generated by airfoil profiles placed in a uniform laminar flow, Journal of Fluid Mechanics 134 (1983) 33-47.

[3] T. P. Chong, P. F. Joseph, M. J. Kingan, An investigation of airfoil tonal noise at different Reynolds numbers and angles of attack, Applied Acoustics 74 (2013) 38-48.

[4] A. McAlpine, E. C. Nash, M. V. Lowson, On the generation of discrete frequency tones by the flow around an airfoil, Journal of Sound and Vibration 222 (1999) 753-779.

[5] M. J. Kingan, J. R. Pearse, Laminar boundary layer instability noise produced by an aerofoil, Journal of Sound and Vibration 322 (2009) 808-828.

[6] C. K. W. Tam, Discrete tones of isolated airfoils, Journal of the Acoustical Society of America 55 (1974) 1173-1177.

[7] G. Desquesnes, M. Terracol, P. Sagaut, Numerical investigation of the tone noise mechanism over laminar airfoils, Journal of Fluid Mechanics 591 (2007) 155-182.

[8] C. K. W. Tam, H. Ju, Airfoil tones at moderate Reynolds number, Journal of Fluid Mechanics 690 (2012) 536-570.

[9] M. V. Lowson, S. P. Fiddes, E. C. Nash, Laminar boundary layer aeroacoustic instabilities, AIAA Paper No. 94-0358, 1994.

[10] E. C. Nash, M. V. Lowson, A. McAlpine, Boundary layer instability noise on airfoils, Journal of Fluid Mechanics 382 (1999) 27-61.

[11] M. S. Howe, Noise produced by a sawtooth trailing edge, Journal of the Acoustical Society of America 90 (1991) 482-487.

[12] T. Dassen, R. Parchen, J. Bruggeman, F. Hagg, Results of a wind tunnel study on the reduction of airfoil self-noise by the application of serrated blade trailing edges, Proceedings of the European Wind Energy Conference, 1996.

[13] S. Oerlemans, M. Fisher, T. Maeder, K. Kogler, Reduction of wind turbine noise using optimized airfoils and trailing-edge serrations, AIAA Journal 47 (2009) 1470-1481. 
[14] M. Gruber, P. F. Joseph, T. P. Chong, On the mechanism of serrated trailing edge noise reduction, AIAA Paper No. 11-2781, 2011.

[15] M. Gad-el-Hak, Flow control: passive, active, and reactive flow management, Cambridge University press, 2000.

[16] T. P. Chong, P. F. Joseph, P. O. A. L. Davies, Design and performance of an open jet wind tunnel for aero-acoustic measurement, Applied Acoustics 70 (2009) 605-614.

[17] T. P. Chong, P. F. Joseph, P. O. A. L. Davies, A parametric study of passive flow control for a short, high area ratio 90 deg curved diffuser, Transaction of ASME: Journal of Fluids Engineering 130 (2008) 111104-1-111104-12.

[18] T. P. Chong, P. F. Joseph, M. Gruber, An experimental study of airfoil instability noise with trailing edge serrations, AIAA Paper No. 10-3723, 2010.

[19] J. P. Stack, S. M. Mangalam, S. A. Berry, A unique measurement technique to study laminarseparation bubble characteristics on an airfoil, AIAA Paper No. 87-1271, 1987.

[20] F. Ida, J. Shinohara, T. Kunikyo, K. Nakasu, S. C. P. Cook, Experimental quantitative turbine boundary-layer investigations using multiple hot film sensor, AIAA Paper No. 94-2537, 1994.

[21] T. Lee, S. Basu, Measurement of unsteady boundary layer developed on an oscillating airfoil using multiple hot-film sensors, Experiments in Fluids 25 (1998) 108-117.

[22] M. Drela, XFOIL: an analysis and design system for low Reynolds Number airfoils, Conference on Low Reynolds Number Airfoil Aerodynamics, University of Notre Dame, 1989.

[23] R. Paterson, P. Vogt, M. Fink, C. Munch, Vortex noise of isolated airfoils, Journal of Aircraft 10 (1973) 296-302.

[24] W. K. Blake, Turbulent boundary-layer wall-pressure fluctuations on smooth and rough walls, Journal of Fluid Mechanics 44 (1970) 637-660.

[25] M. Goody, Empirical spectral model of surface pressure fluctuations, AIAA Journal 42 (2004) 1788-1794.

[26] T. P. Chong, A. Vathylakis, P. F. Joseph, M. Gruber, Self noise produced by an airfoil with nonflat plate trailing edge serrations, AIAA Journal, 51 (2013) 2665-2677.

[27] T. P. Chong, P. F. Joseph, "Ladder" structure in tonal noise generated by laminar flow around an airfoil, Journal of the Acoustical Society of America 131 (2012) EL461-EL467.

[28] J. C. Yu, C. K. W. Tam, Experimental investigation of the trailing edge noise mechanism, AIAA Journal 16 (1977) 1046-1052.

[29] G. E. Mattingly, W. O. Criminale, The stability of an incompressible two-dimensional wake, Journal of Fluid Mechanics 51 (1972) 233-272.

[30] W. Koch, Local instability characteristics and frequency determination of self-excited wake flows, Journal of Sound and Vibration 99(1) (1985) 53-83.

[31] B. M. Woodley, N. Peake, Global linear stability analysis of thin aerofoil wakes, Journal of Fluid Mechanics 339 (1997) 239-260. 
[32] M. Turkyilmazoglu, J. S. B. Gajjar, A. I. Ruban, The absolute instability of thin wakes in an incompressible / compressible fluid, Theoretical and Computational Fluid Dynamics 13 (1999) 91-114.

[33] T. P. Chong, P. F. Joseph, An experimental study of tonal noise mechanisms of laminar airfoils, AIAA Paper No. 09-3345, 2009. 
Model A

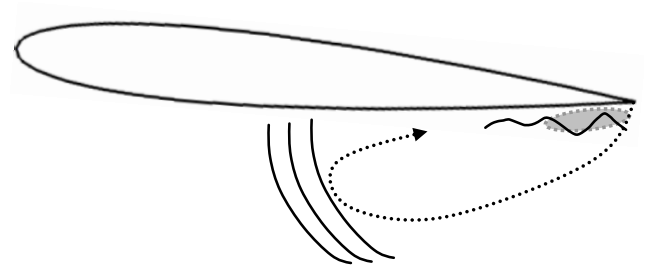

Model C

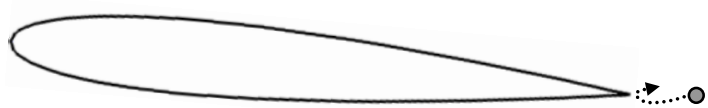

\section{Model B}

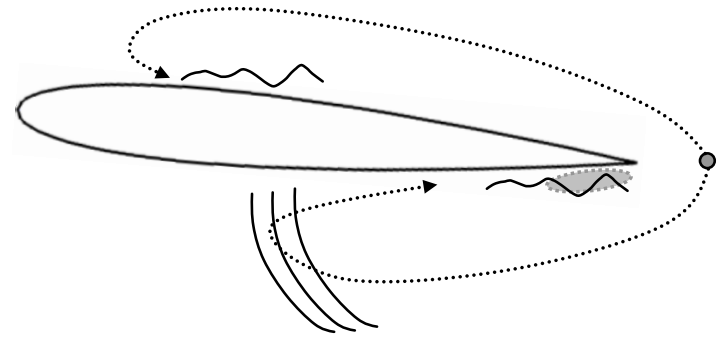

- Dipole noise source (wake)

T-S wave

$\ldots$ Separation bubble

(II Acoustic wave

- Loop structure

Fig. 1 Several aeroacoustic feedback models proposed in the literature for the generation of airfoil tonal noise. Note that Model A is based on [2], Model B is based on [7] and Model $\mathrm{C}$ is based on [6]. 

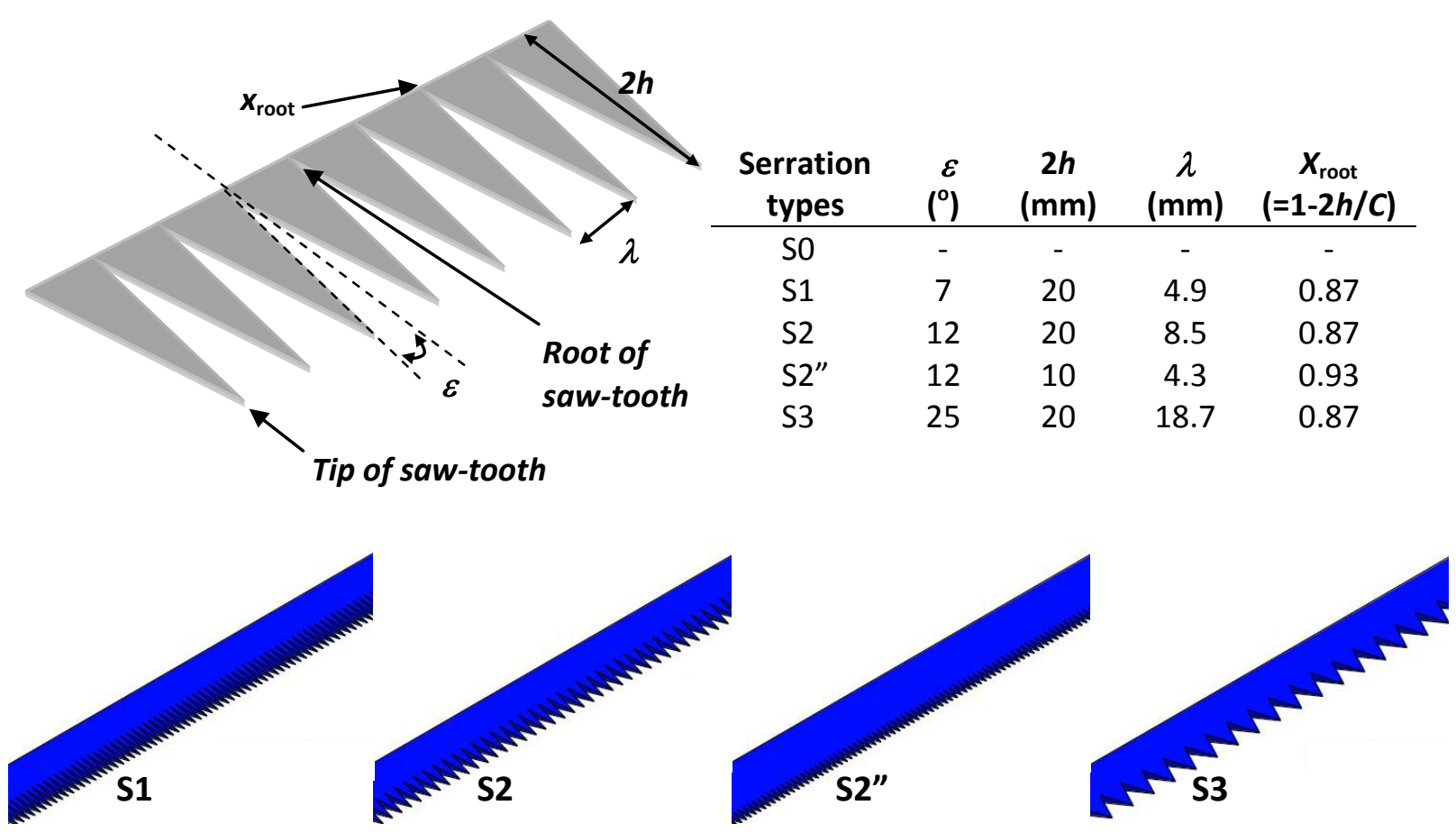

Fig. 2 Parameters associated with a saw-tooth serration at the airfoil's trailing edge. The table summarizes the parameters associated with all of the serration cases (S1, S2, S2" and S3) tested in this study. Note that SO refers to a straight trailing edge. The retained geometries of the trailing edge serrations are illustrated in the scaled 3D drawings at the bottom. 


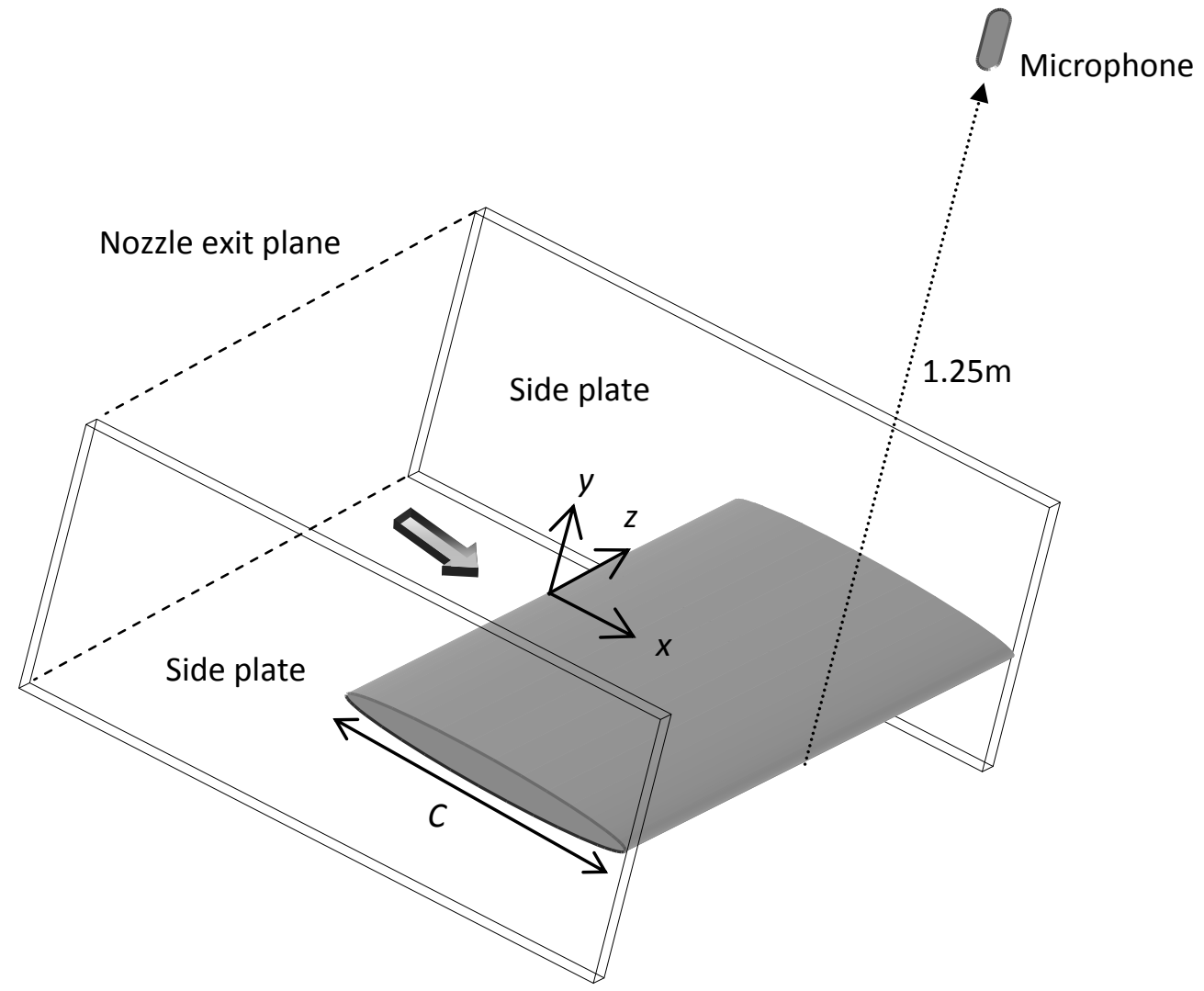

Fig. 3 Experimental set up of the free field tests performed in the anechoic chamber. The far-field microphone is placed at $1.25 \mathrm{~m}$ from the trailing edge of the airfoil at a polar angle of $\zeta=90^{\circ}$ to the horizontal axis. 


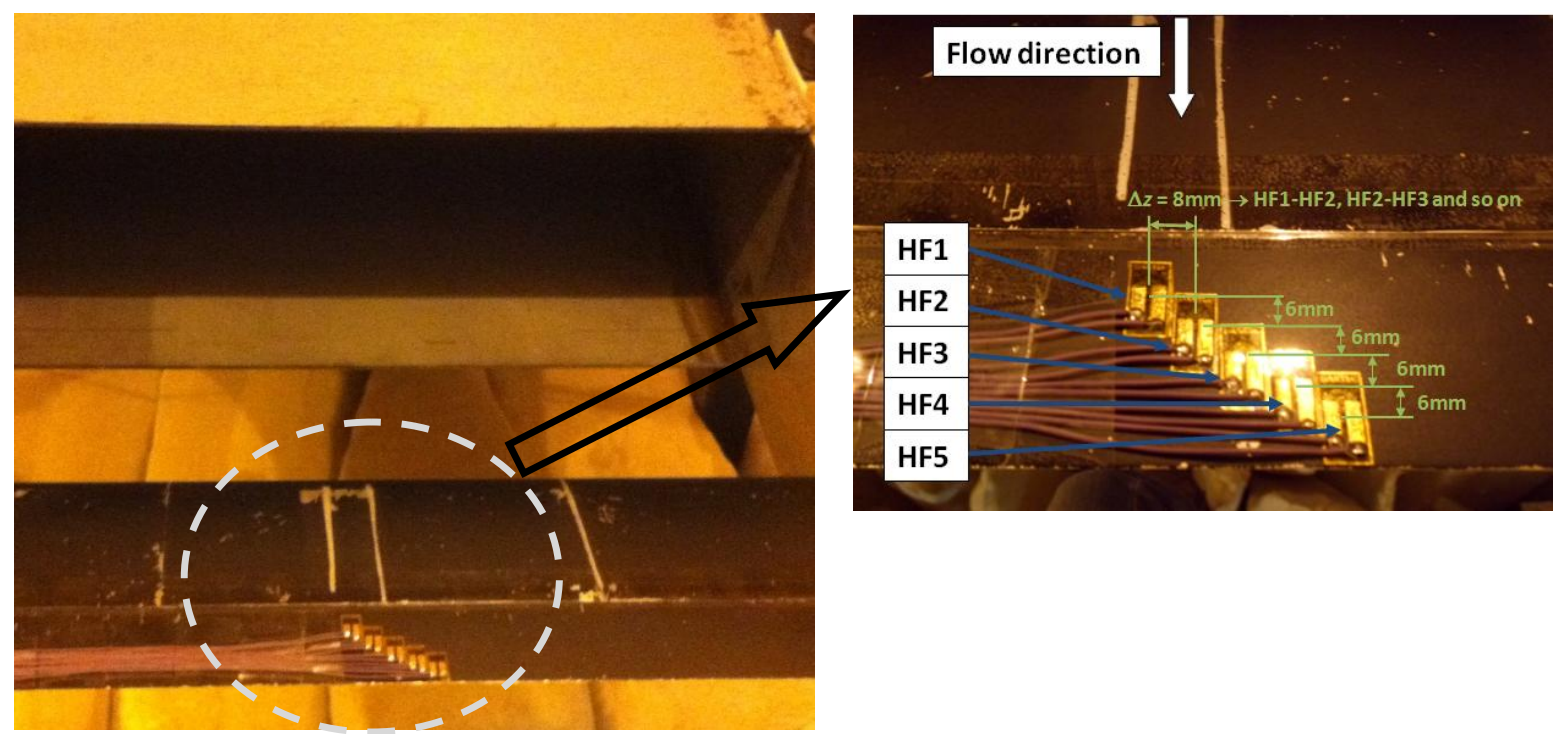

Fig. 4 Arrangement of the surface mounted hot-film arrays used in this study for the detection of the separation point near the airfoil's trailing edge. Note that the experiment was performed in the ISVR open jet wind tunnel using the same set-up as the acoustic measurements as shown in Fig. 3. 


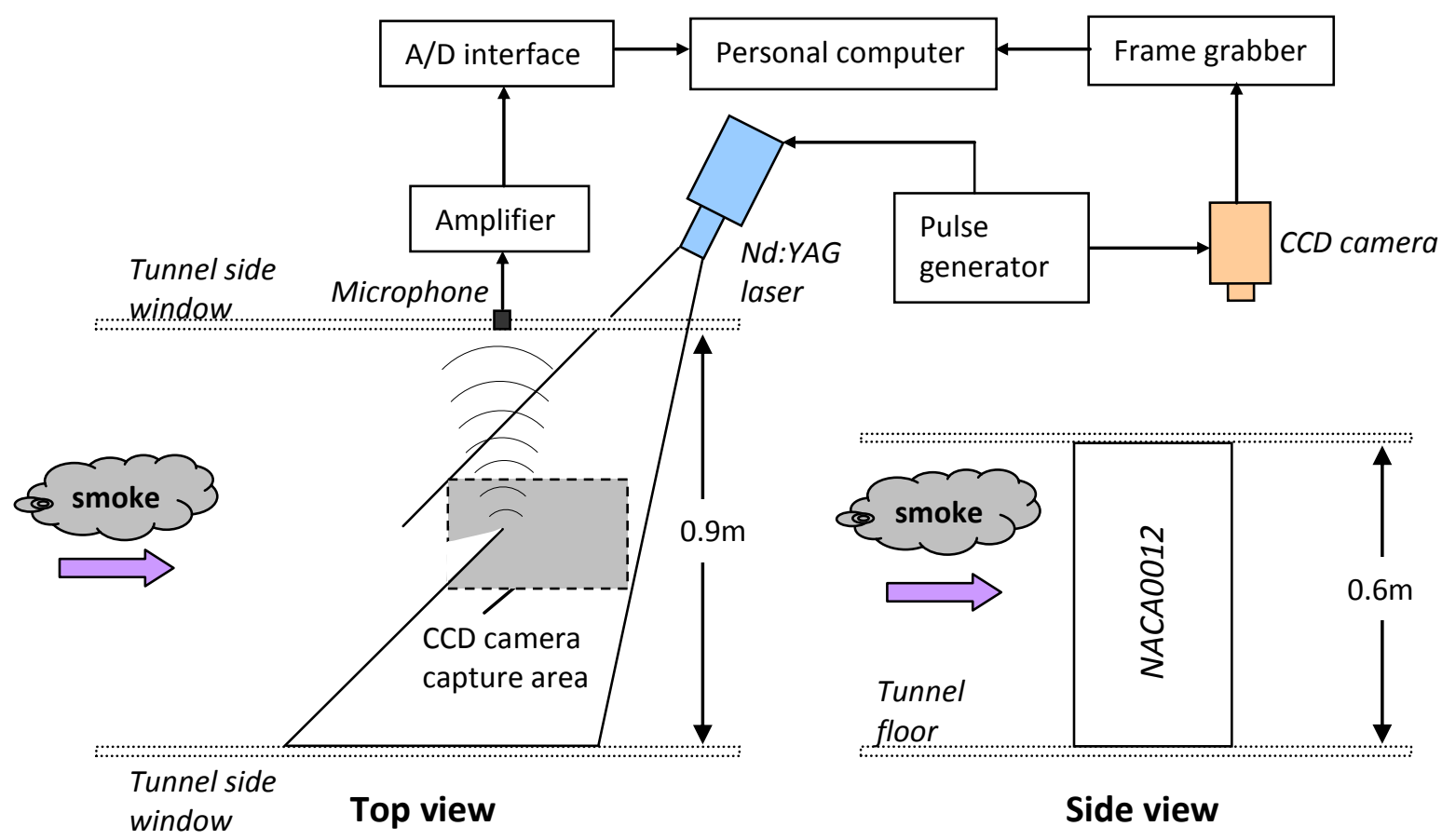

Fig. 5 The PIV and microphone set-up for the aerodynamic/acoustic measurements in the closed-working section wind tunnel. 
(a)

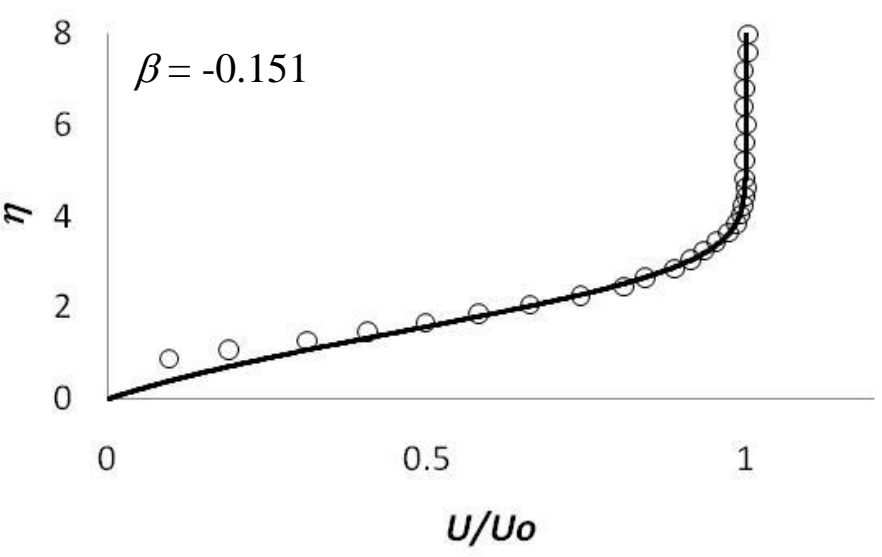

(b)

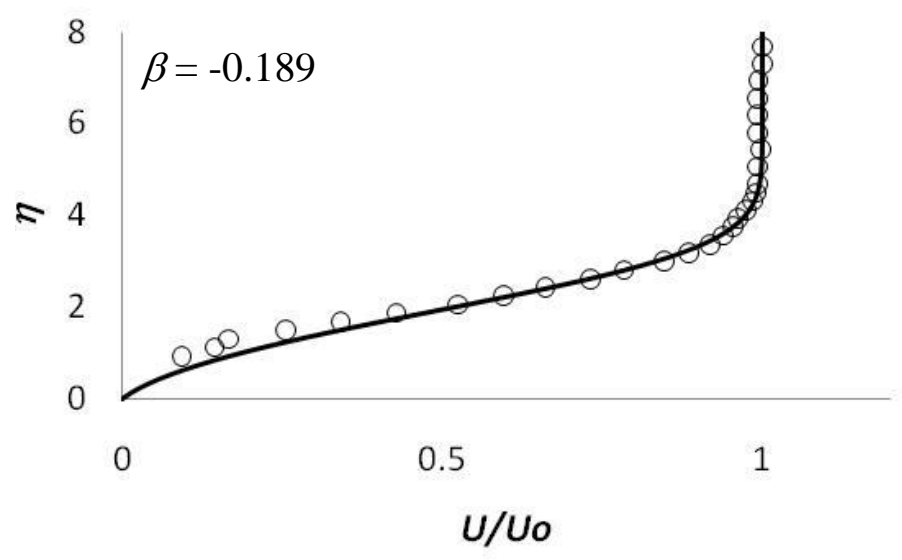

(c)

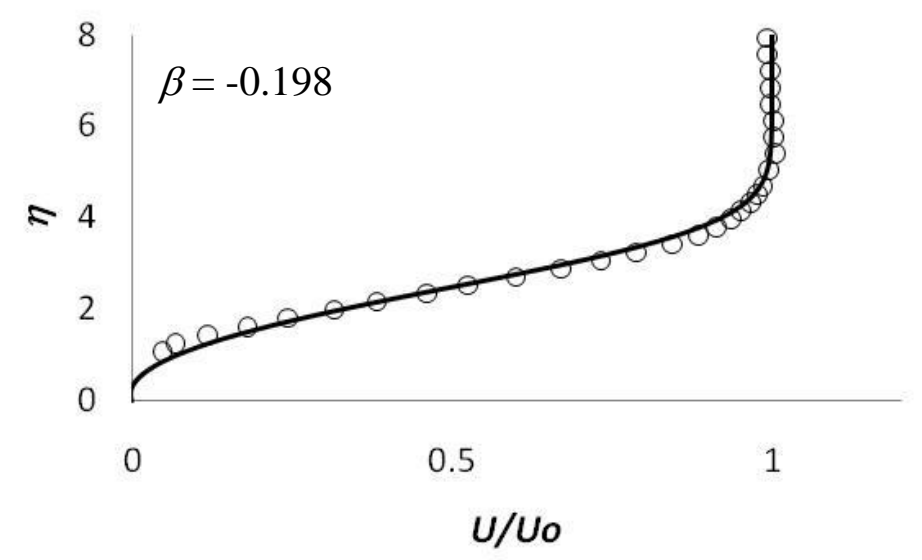

Fig. 6 Comparisons of the Falkner Skan velocity profile $(-)$ and the measured velocity profile $U / U_{o}(\circ)$ at the airfoil's pressure surface at $\theta=5^{\circ}$ and freestream velocity of $15 \mathrm{~ms}^{-1}$ : (a) $x / C=0.80$; (b) $x / C=0.87$; and (c) $x / C=0.93$. Note that $U_{o}$ is the freestream flow velocity. The trailing edge in this case is the straight edge type (S0). 
(a)

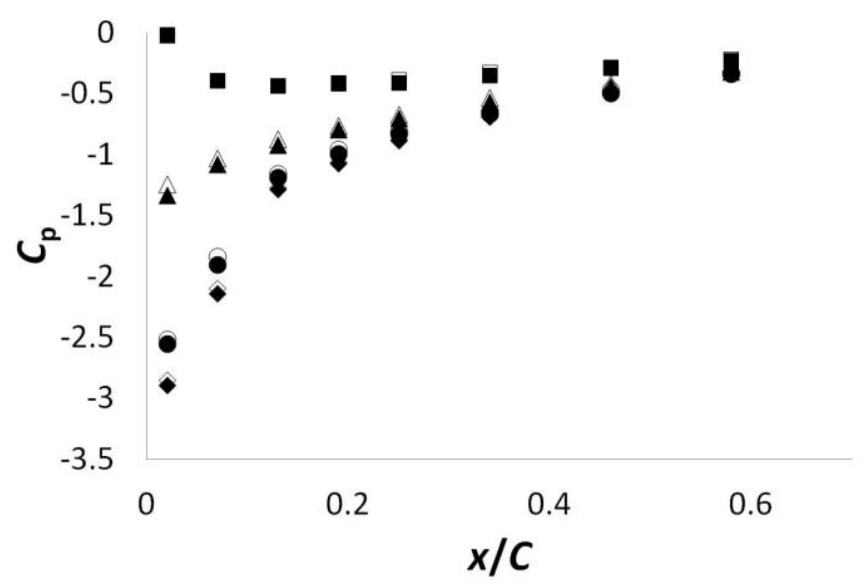

(b)

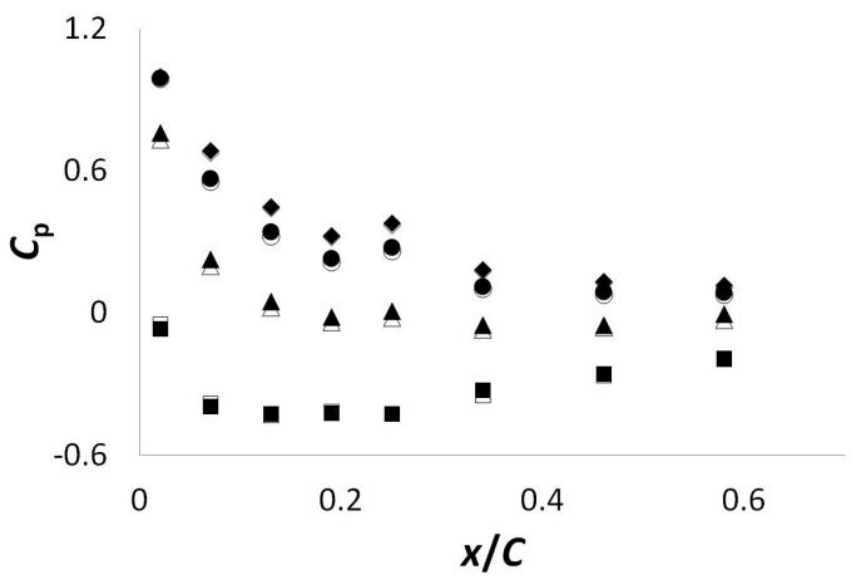

Fig. 7 Comparison of the pressure coefficient $C_{\mathrm{p}}$ at $0.02<x / C<0.58$, at the (a) suction surface; and (b) pressure surface, when the NACA-0012 is installed with either S0 or S3 trailing edges. Summary of symbols: $\theta=0^{\circ}$ ( $\mathrm{S} 0 \square, \mathrm{S} 3 \square$ ), $\theta=4^{\mathrm{O}}$ (S0 $\boldsymbol{\Delta}$, $\mathrm{S} 3 \Delta), \theta=8^{\circ}(\mathrm{S} 0 \bullet, \mathrm{S} 3 \circ)$ and $\theta=10^{\circ}(\mathrm{S} 0 \bullet, \mathrm{S} 3 \diamond)$. 


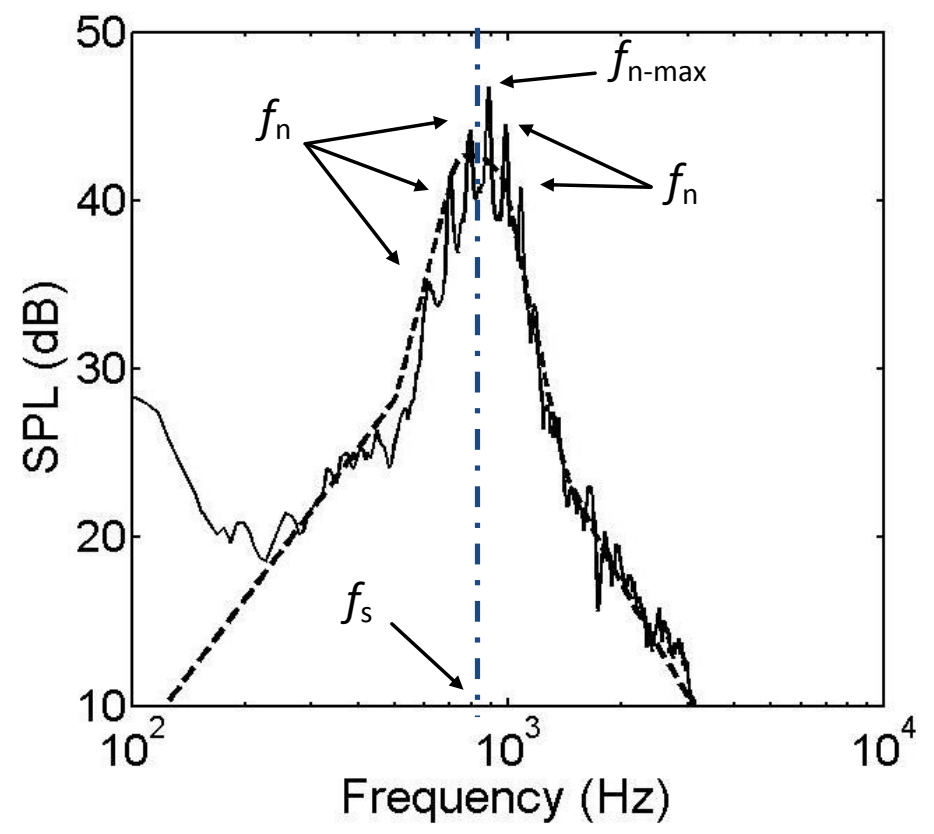

Fig. 8 Examples of a measured $(-$ laminar tonal noise spectrum (ref.20 $\mu \mathrm{Pa}, 1 \mathrm{~Hz}$ bandwidth) and the corresponding predicted noise spectrum (-- ) by Brooks et al. [1]. Note that the predicted level has been adjusted from the original 1/3-octave bandwidth to a level compatible with narrowband of $1 \mathrm{~Hz}$ bandwidth. 
(a)

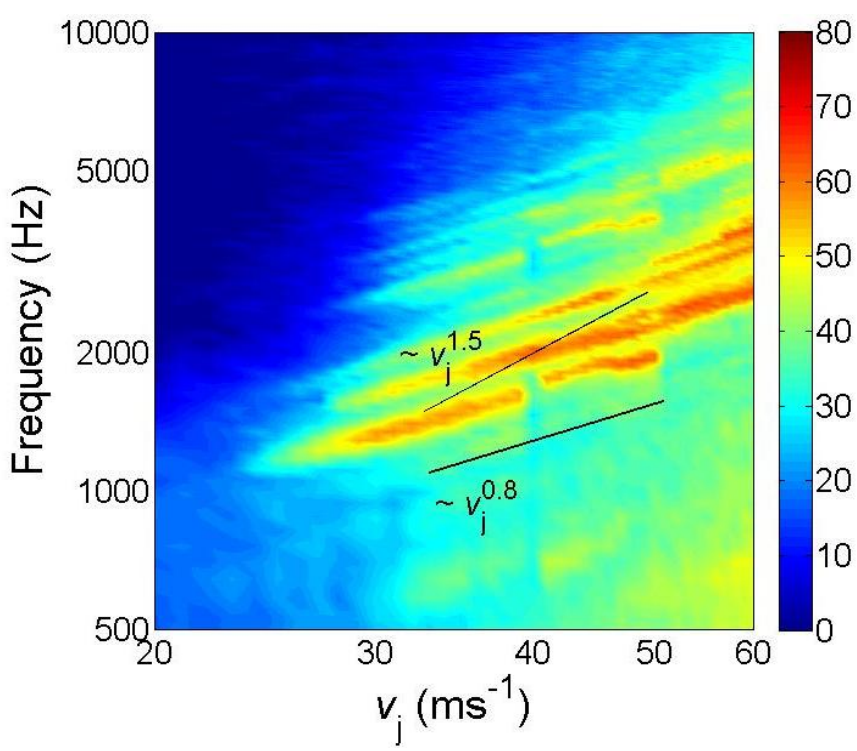

(c)

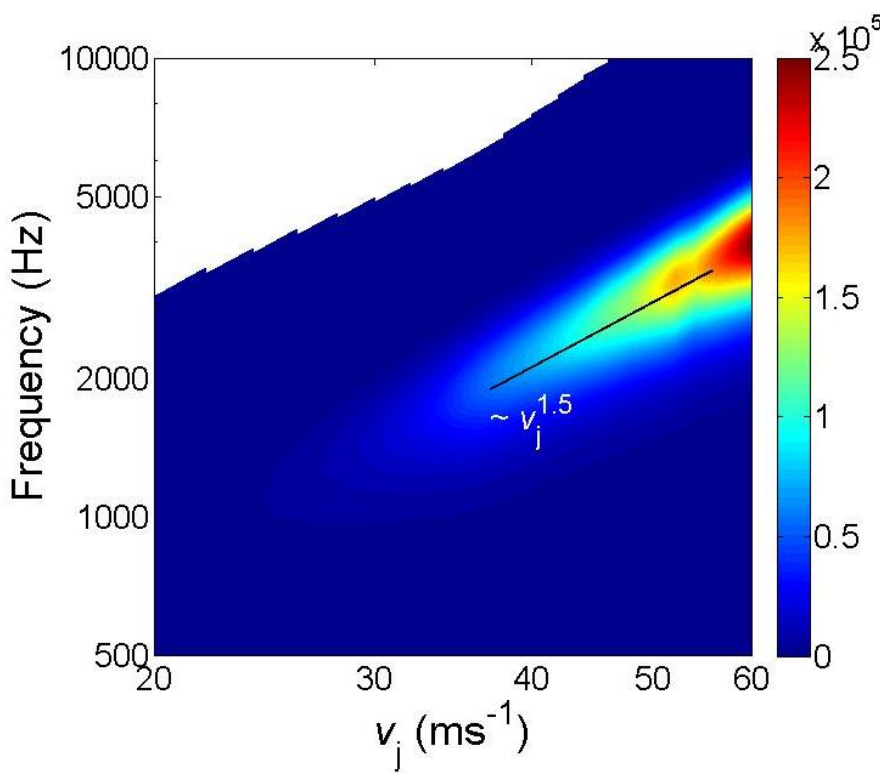

(b)

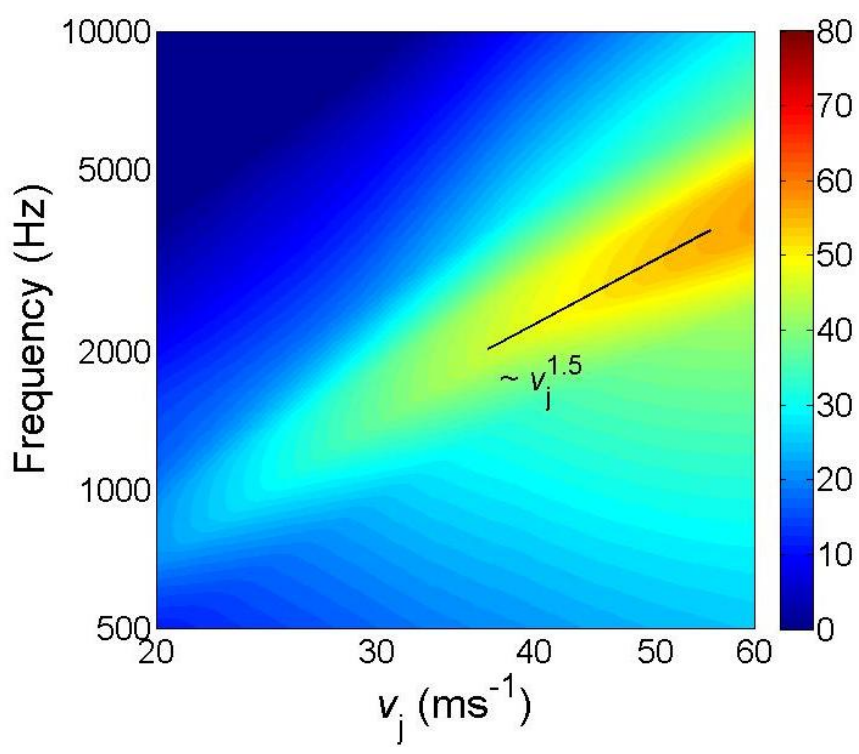

(d)

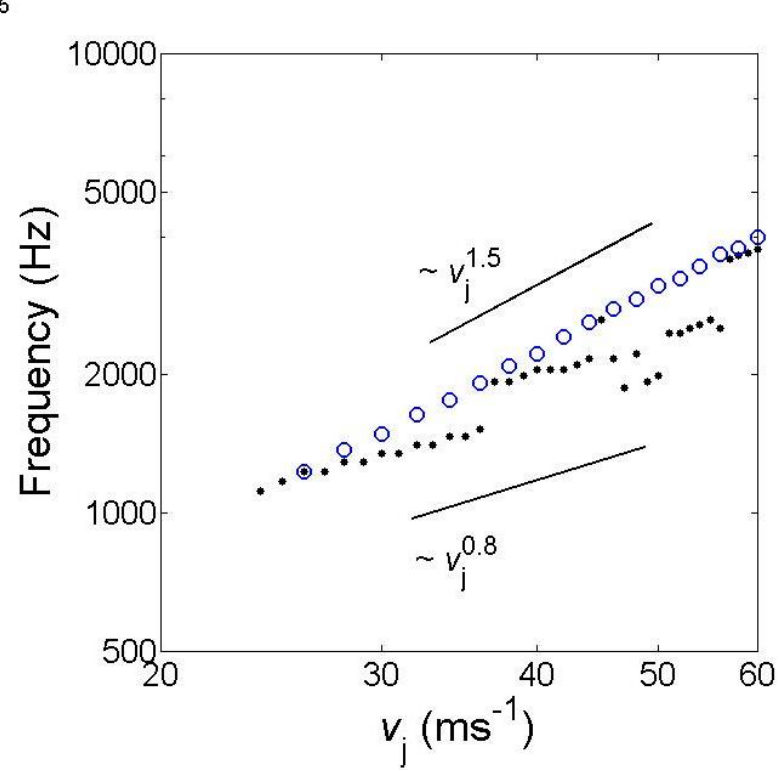

Fig. 9 Frequency-velocity colormaps at $\theta=4.2^{\circ}$ for (a) sound pressure level, $\mathrm{dB}$ (ref $20 \mu \mathrm{Pa}$ ) with $1 \mathrm{~Hz}$ bandwidth for the radiated noise; (b) equivalent sound pressure level, $\mathrm{dB}$, predicted by Brooks et al.'s model; (c) T-S wave total amplification at the pressure surface; and (d) frequencies correspond to the highest instability tonal noise amplitude $\left(f_{\text {n-max }},, \bullet\right)$ and the maximum T-S wave amplification factor $\left(f_{\mathrm{TS}-\mathrm{max}}, \mathrm{O}\right)$ at the pressure surface. 

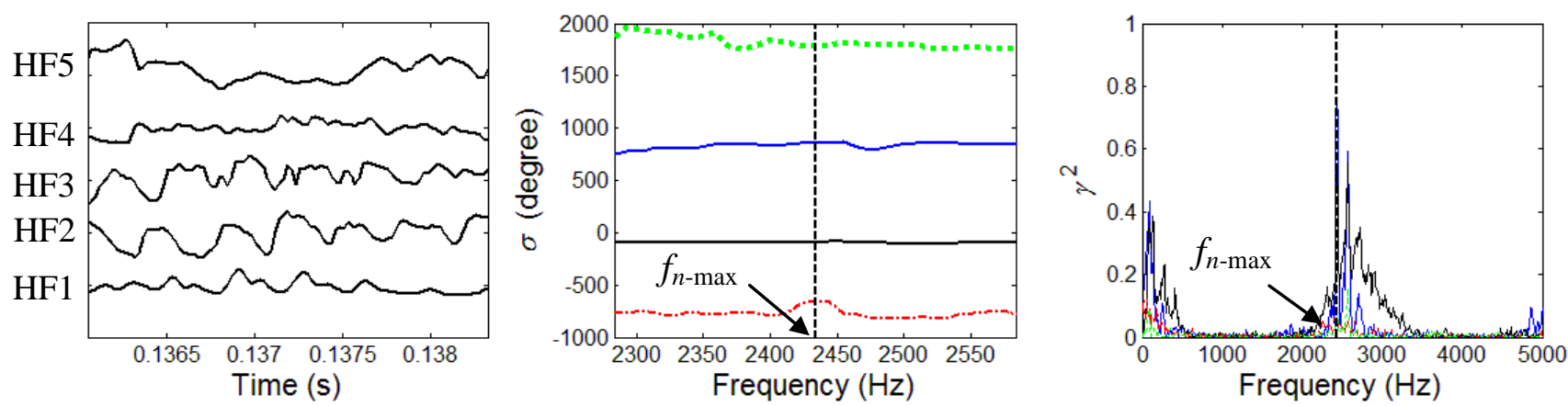

Fig. 10 Simultaneously measured raw signals and their associated phase angles $(\sigma)$ and coherences $\left(\gamma^{2}\right)$ obtained by the surface mounted hot film arrays at $\theta=4.2^{\circ}$ and $v_{\mathrm{j}}=$ 40ms ${ }^{-1}$. HF1-HF2 (blue -), HF2-HF3 (black -), HF3-HF4 (red -.-) and HF4-HF5 (green ….) for the phase angle and coherence spectral diagrams. 


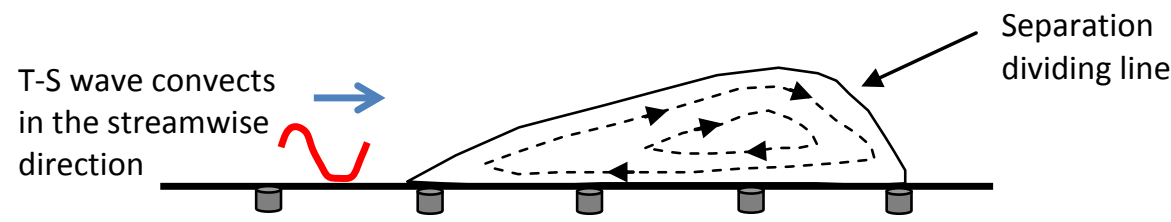

T-S wave detaches from the wall and interacts with the separating shear layer
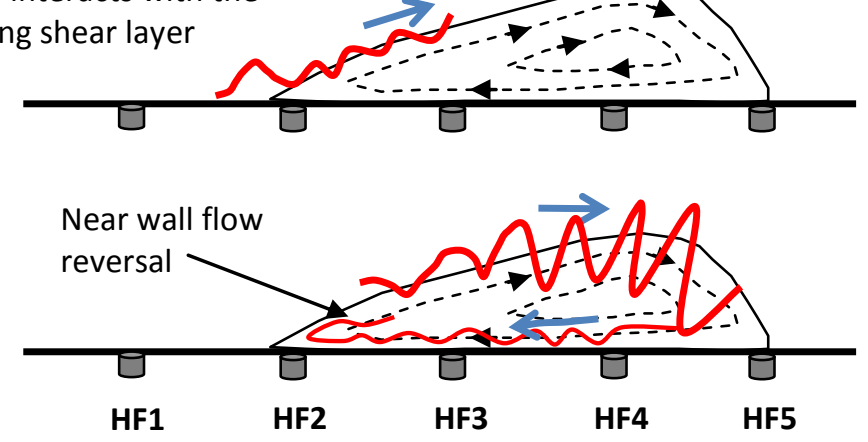

Fig. 11 Idealized schematics which show the interaction between a convecting T-S wave and a "stationary" separation bubble. Drawings are not to scale. 
(a)

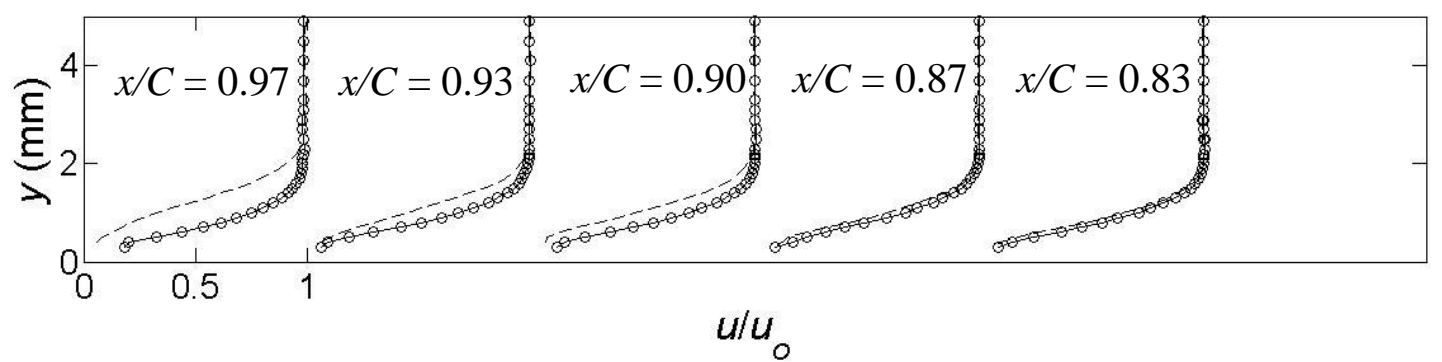

(b)

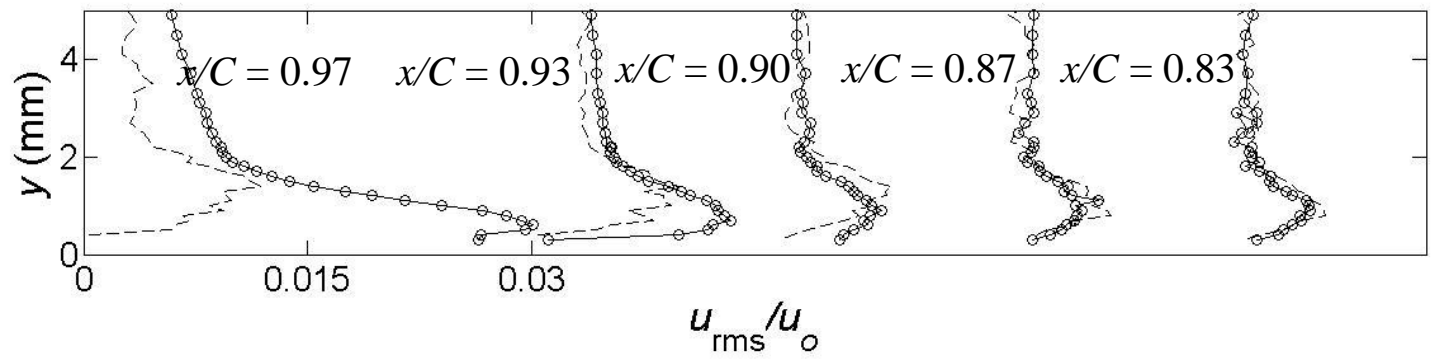

Fig. 12 Streamwise distributions $\left(x / C=0.83,0.87\left(X_{\text {root }}\right), 0.90,0.93\right.$ and 0.97$)$ of the (a) mean velocity boundary layer profiles $\left(u / U_{o}\right)$; and (b) rms velocity fluctuation boundary layer profiles $\left(u_{\mathrm{rms}} / U_{o}\right)$ at the airfoil's pressure surface with $\theta=5^{\circ}$ and the freestream velocity sets at $15 \mathrm{~ms}^{-1}$. The comparisons are made between the straight trailing edge S0 (-- ) and the serrated trailing edge S3 (-o-). 

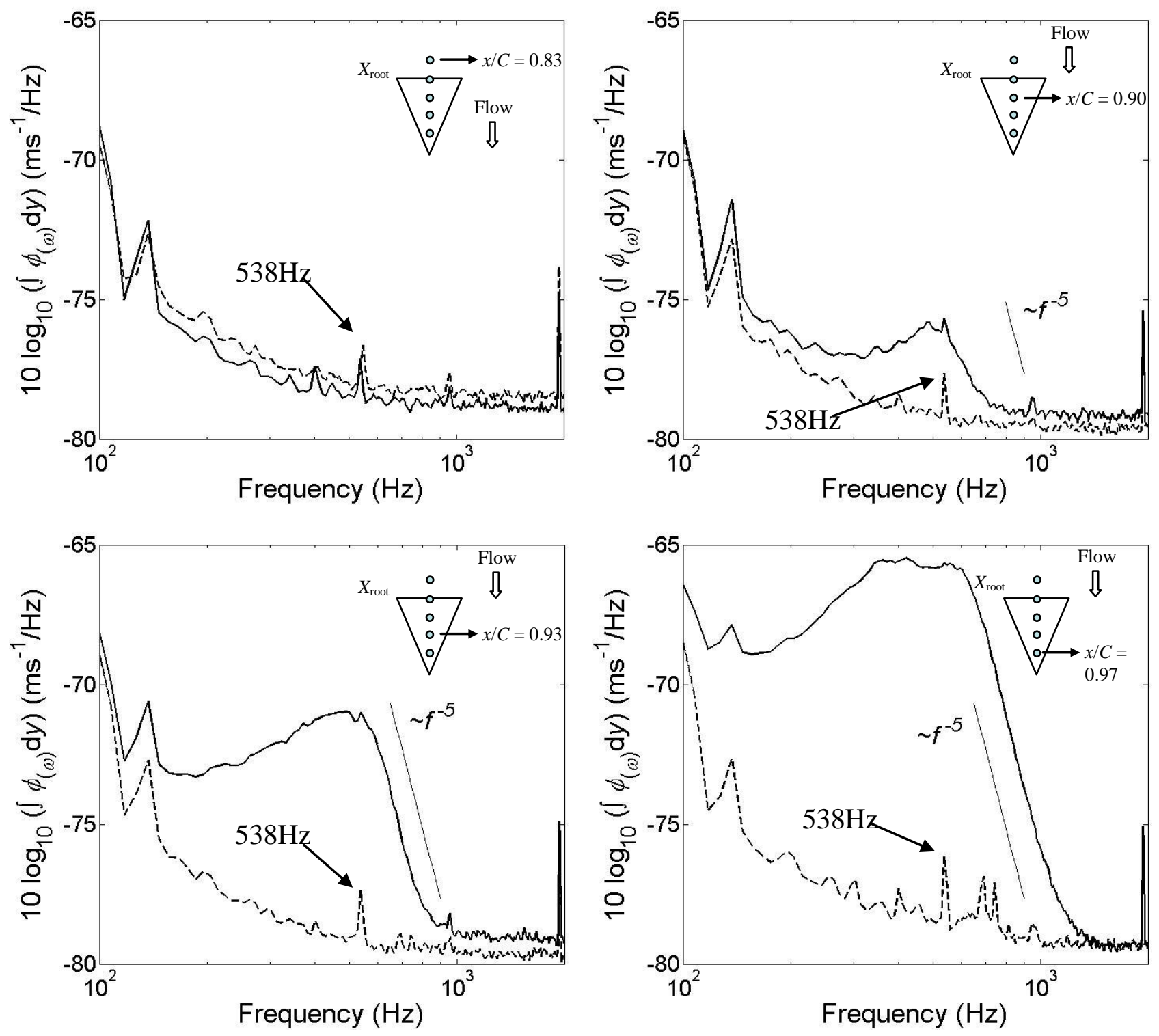

Fig. 13 Streamwise developments of the integrated boundary layer velocity spectra from $x / C=0.83$ to $x / C=0.97$ for the straight $\mathrm{S} 0(---)$ and serrated S3 (-) cases at the airfoil's pressure surface with $\theta=5^{\circ}$ and the freestream velocity at $15 \mathrm{~ms}^{-1}$. 
(a)

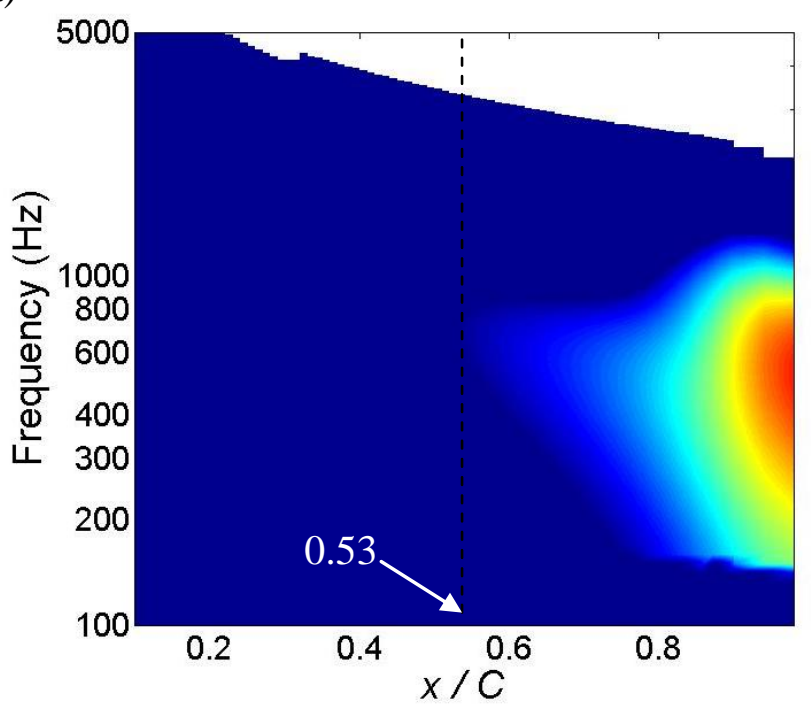

(b)

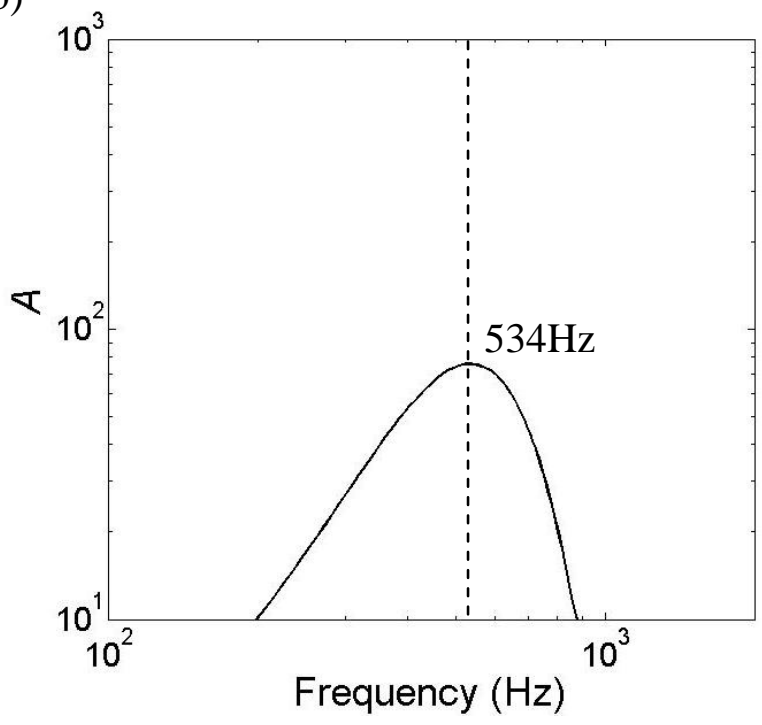

Fig. 14(a) Streamwise distribution of the T-S wave amplification growth rate $\left(-\alpha_{\mathrm{i}}\right)$; and (b) T-S wave total amplification $A$ developed at the airfoil's pressure surface for the S0 case at $\theta=$ $5^{\circ}$ and freestream velocity at $15 \mathrm{~ms}^{-1}$. 
(a)

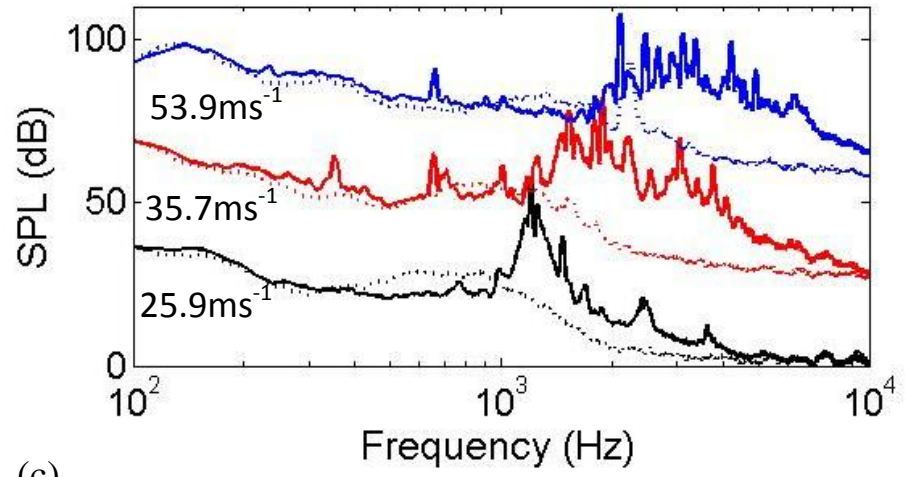

(c)

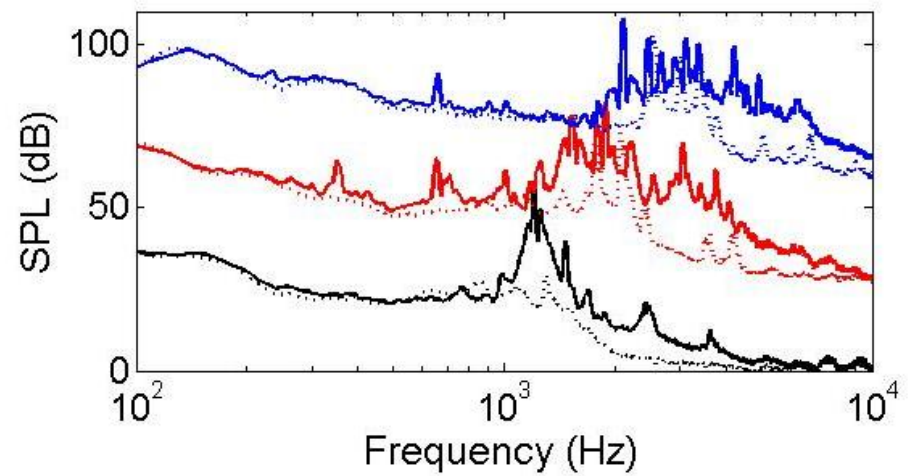

(b)

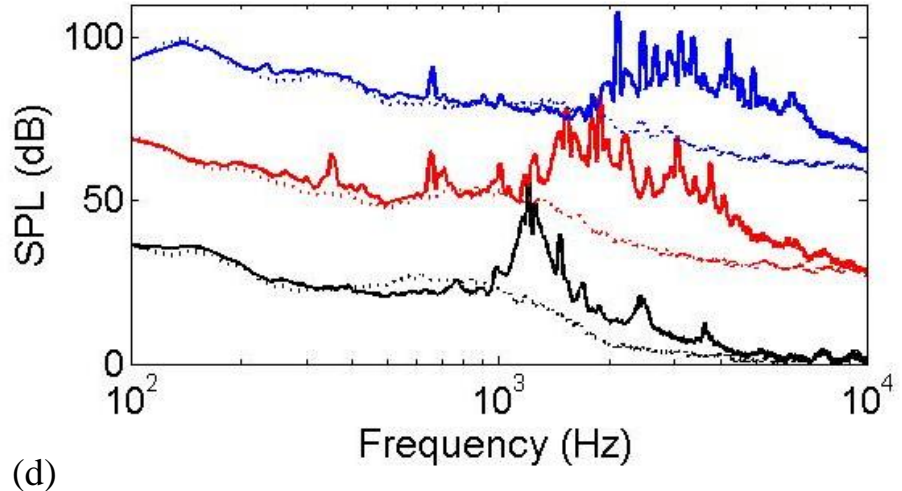

(d)

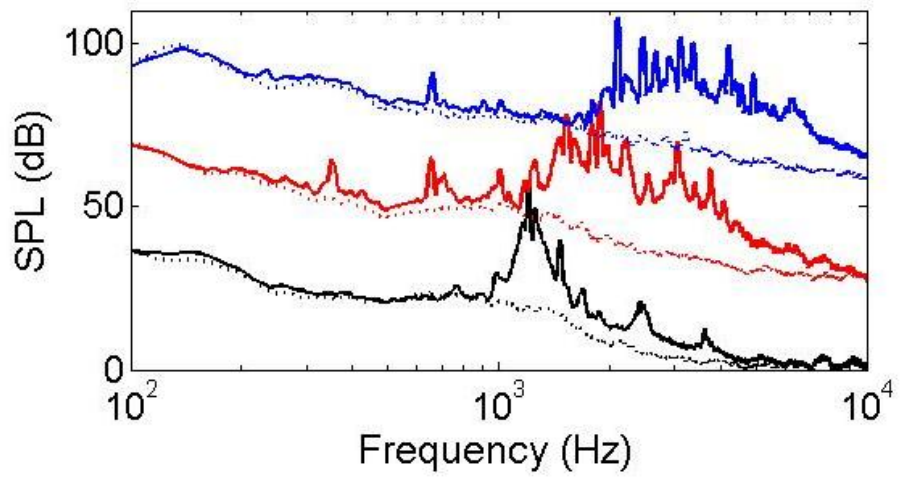

Fig. 15 Airfoil self-noise spectra (ref. $20 \mu \mathrm{Pa}$ with $1 \mathrm{~Hz}$ bandwidth) measured at $\theta=4.2^{\circ}$. Comparisons were made between a straight (solid line) and a serrated (dash line) trailing edge of the following types: (a) S1; (b) S2; (c) S2"; and (d) S3. Each sub-figure contains three jet velocities: $v_{\mathrm{j}}=25.9 \mathrm{~ms}^{-1}$, $35.7 \mathrm{~ms}^{-1}$ and $53.9 \mathrm{~ms}^{-1}$. 
(a)

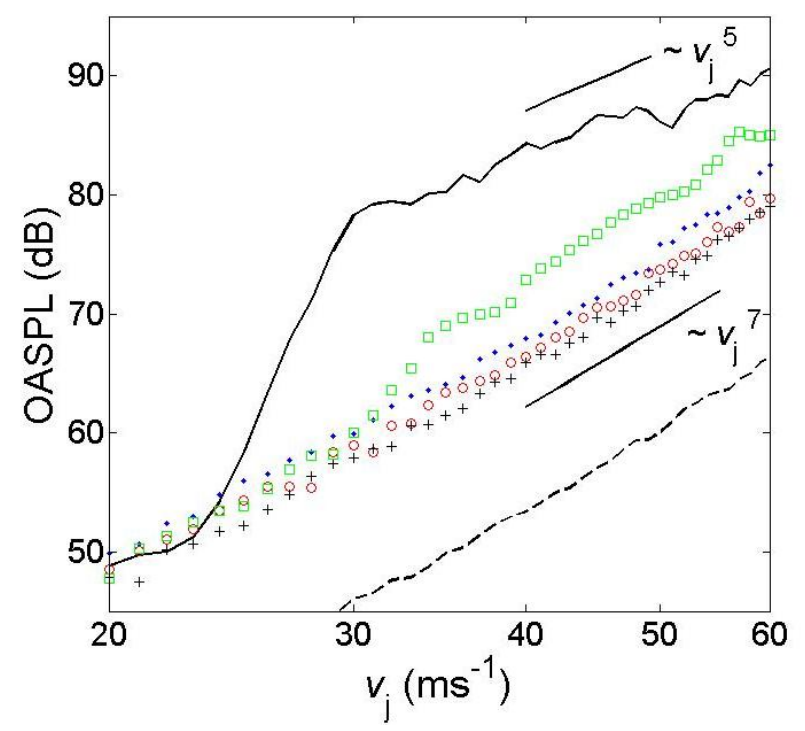

(b)

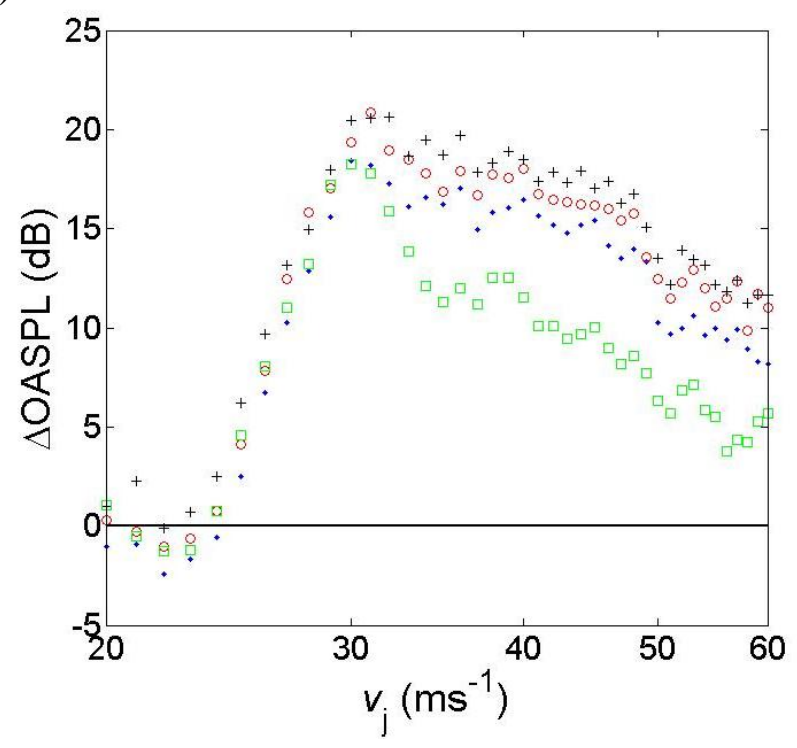

Fig. 16(a) Variations of the OASPL with flow velocity produced by the straight baseline trailing edge: S0 (-) and serrated trailing edges: S1 (॰), S2 (o), S2" ( $\square$ ) and S3 (+). The background noise is also shown (-- ); and (b) Variations of the $\triangle \mathrm{OASPL}$ with flow velocity for the serrated trailing edges: S1 (॰), S2 (o), S2" ( $\square$ ) and S3 (+). Note that $+\Delta$ OASPL values represent noise reduction, and vice versa. 


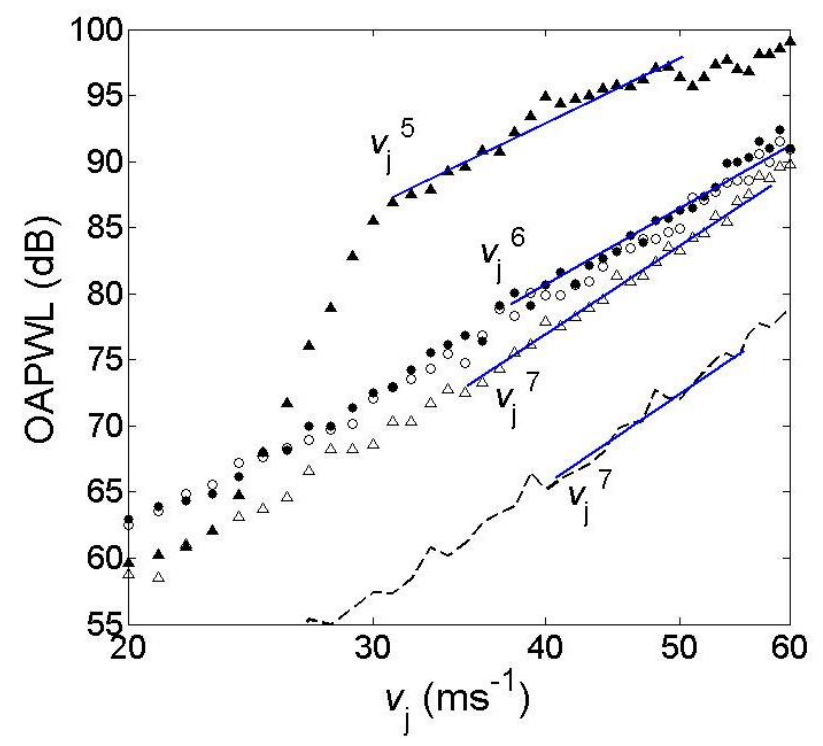

Fig. 17Variations of the OAPWL (ref. $10^{-12} \mathrm{~W}$ ) with flow velocity produced in Case A by: S0 straight trailing edge $(\bullet)$ and S3 serrated trailing edge $(\circ)$; as well as in Case B by: S0 straight trailing edge $(\boldsymbol{\Delta})$ and S3 serrated trailing edge $(\Delta)$. Wind tunnel background noise $(---)$ is also shown. Several solid lines (blue - ), which represent velocity power laws of 5-7, are fitted to the noise data accordingly. 


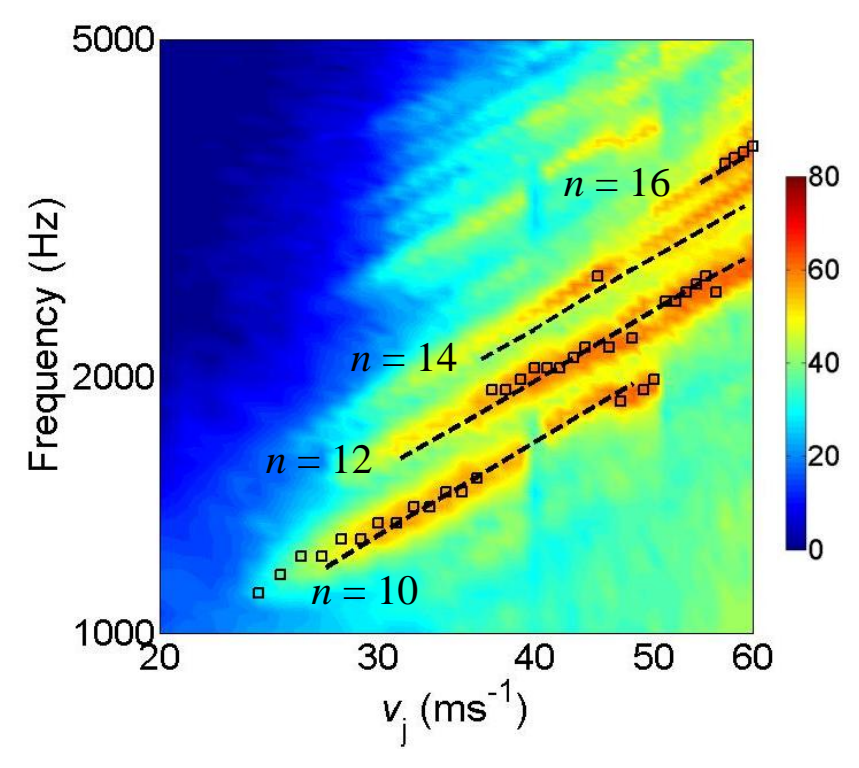

Fig. 18 SPL (dB, ref. $20 \mu \mathrm{Pa}$ with a $1 \mathrm{~Hz}$ bandwidth) contour as a function of frequency and flow velocity for the S0 straight baseline trailing edge at $\theta=$ $4.2^{\circ}$. Frequencies correspond to the largest tone amplitudes $\left(f_{\mathrm{n}-\max }\right)$ at each flow velocity are represented by $(\square)$. The theoretical discrete frequencies $\left(f_{\mathrm{n}}\right)$, calculated by Eqs. (11-12) for $n=10,12,14$ and 16, are represented by $(---)$. 
(a)

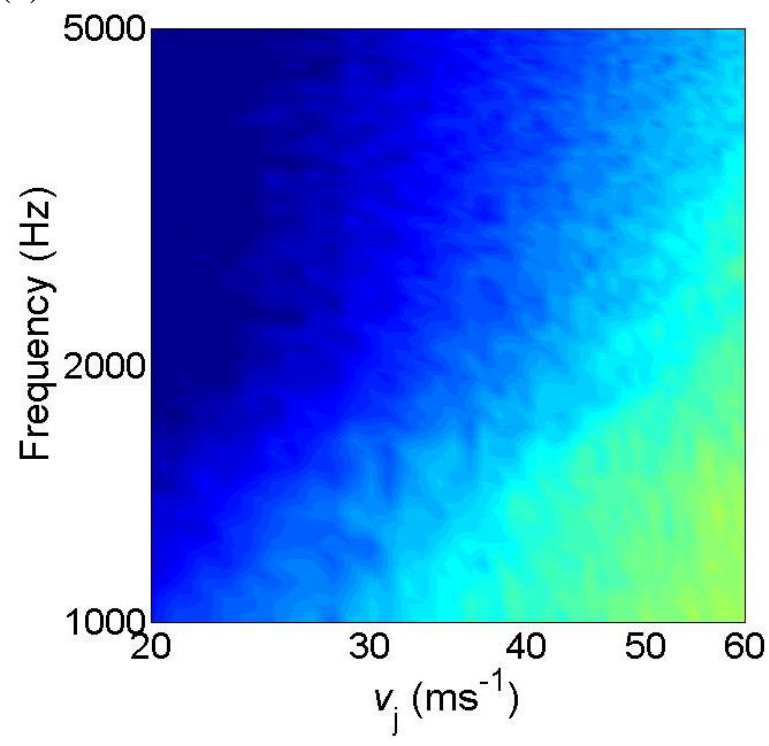

(b)

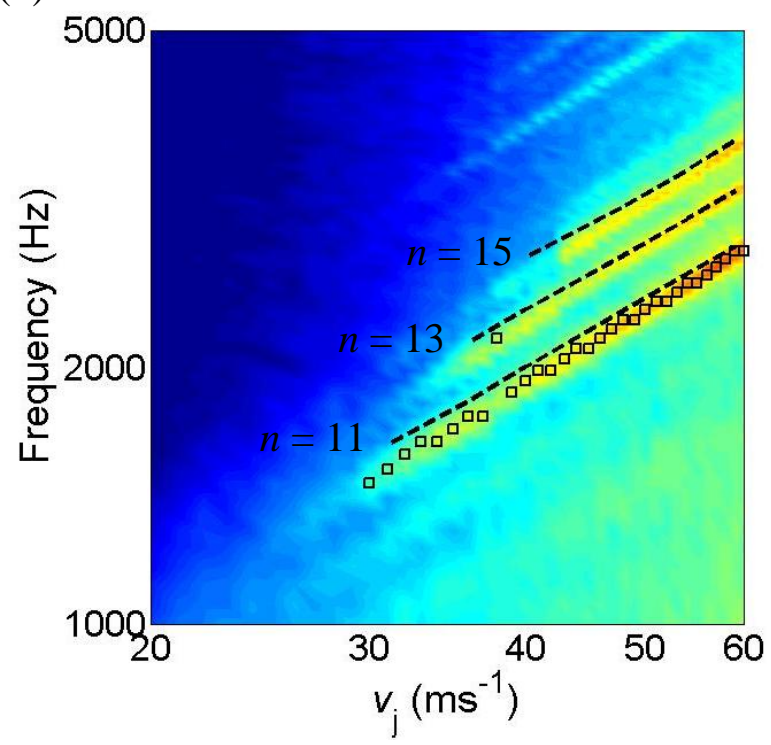

Fig. 19SPL (dB, ref. $20 \mu \mathrm{Pa}$ with a $1 \mathrm{~Hz}$ bandwidth) contours as a function of frequency and flow velocity for the (a) S2; and (b) S2" serrated trailing edges at $\theta=4.2^{\circ}$. No acoustic rung is present for the $\mathrm{S} 2$ case in (a). For the S2" case in (b), frequencies correspond to the largest tone amplitudes $\left(f_{\mathrm{n} \text { - }}\right.$ $\max )$ at each flow velocity are represented by $(\square)$. The theoretical discrete frequencies $\left(f_{\mathrm{n}}\right)$, calculated by Eqs. (11-12) for $n=11,13$ and 15, are represented by (-- $)$. 
(a)

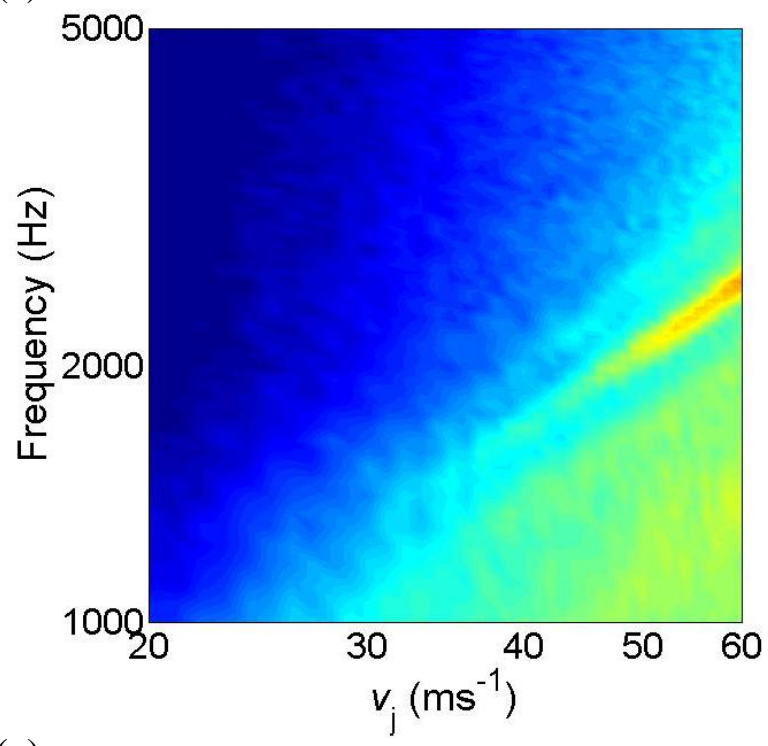

(c)

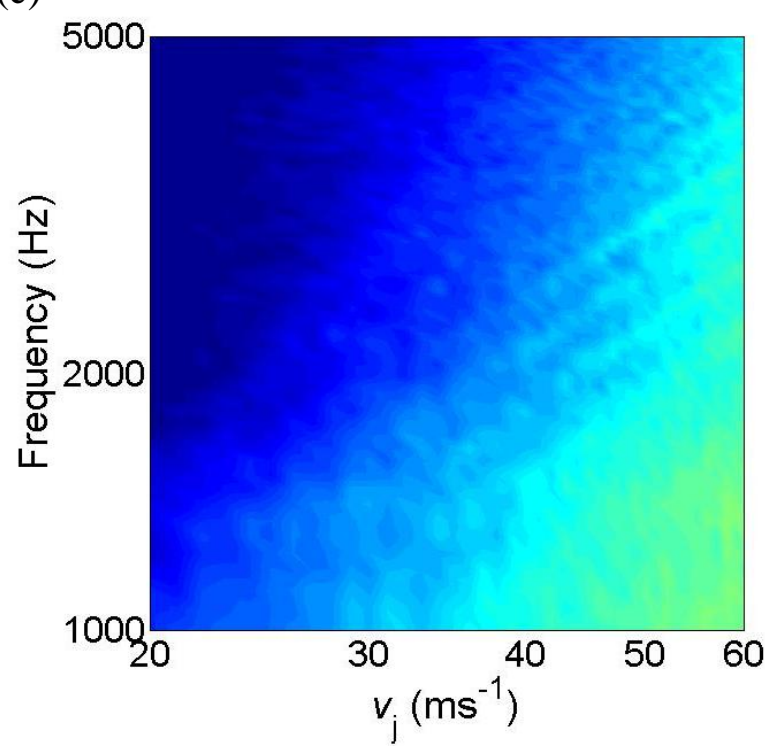

(b)

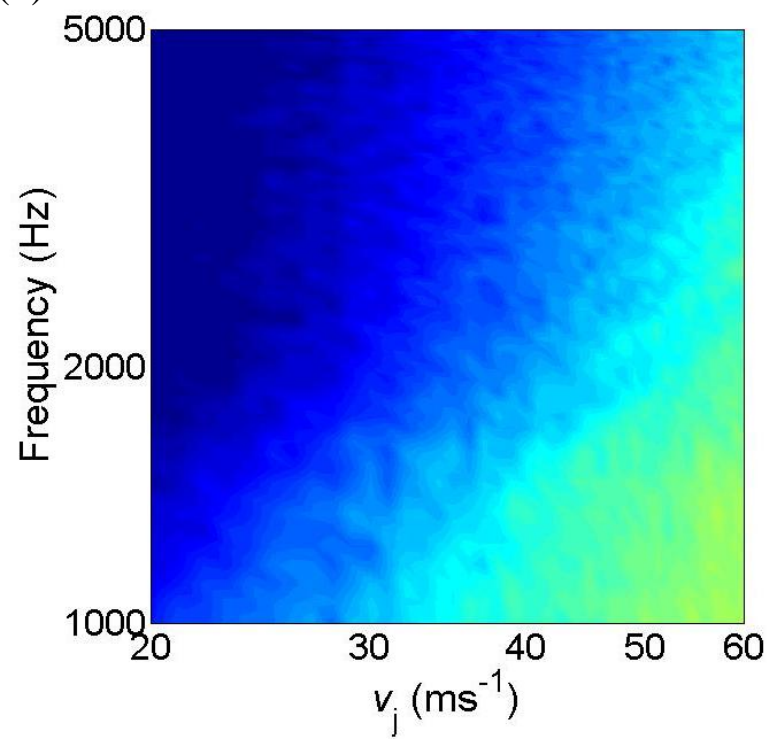

Fig. 20 SPL (dB, ref. $20 \mu \mathrm{Pa}$ with a $1 \mathrm{~Hz}$ bandwidth) contours as a function of frequency and flow velocity for the (a) S1; (b) S2; and (c) S3 serrated trailing edges at $\theta=4.2^{\circ}$. No acoustic rung is present for the S2 and S3 cases in (b) and (c) respectively. For the $\mathrm{S} 1$ case in (a), only a single rung is present. 
$\Longrightarrow$ Flow speed increases in this direction

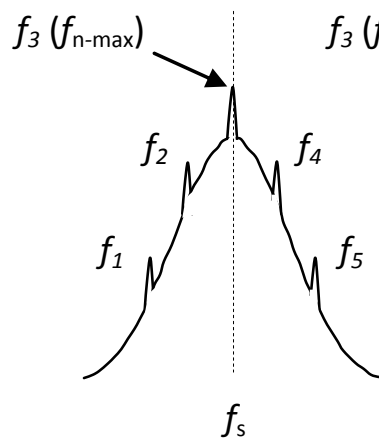

(I)

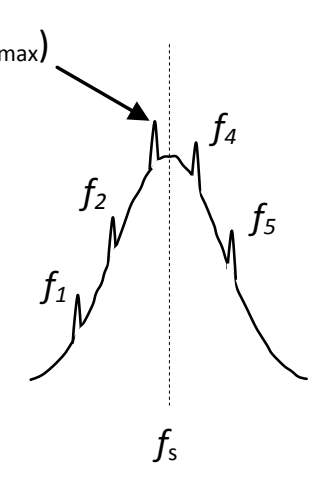

(II)

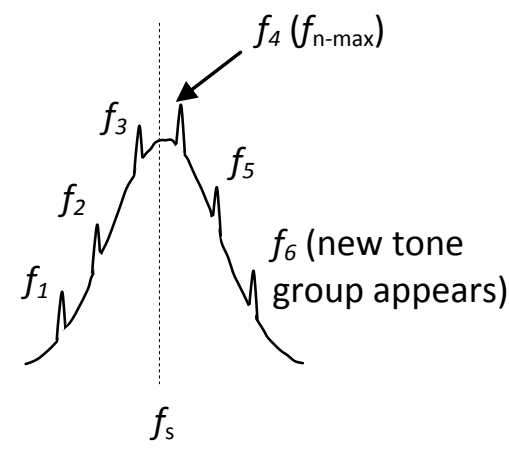

(III)

Fig. 21 Three idealized sketches that show the variations of the broadband hump frequency $\left(f_{\mathrm{s}}\right)$ with respect to the several discrete tone frequencies $\left(f_{n}\right.$, where $n=1,2,3$ and so on) when the flow velocity increases from left to right [27]. 
(a)

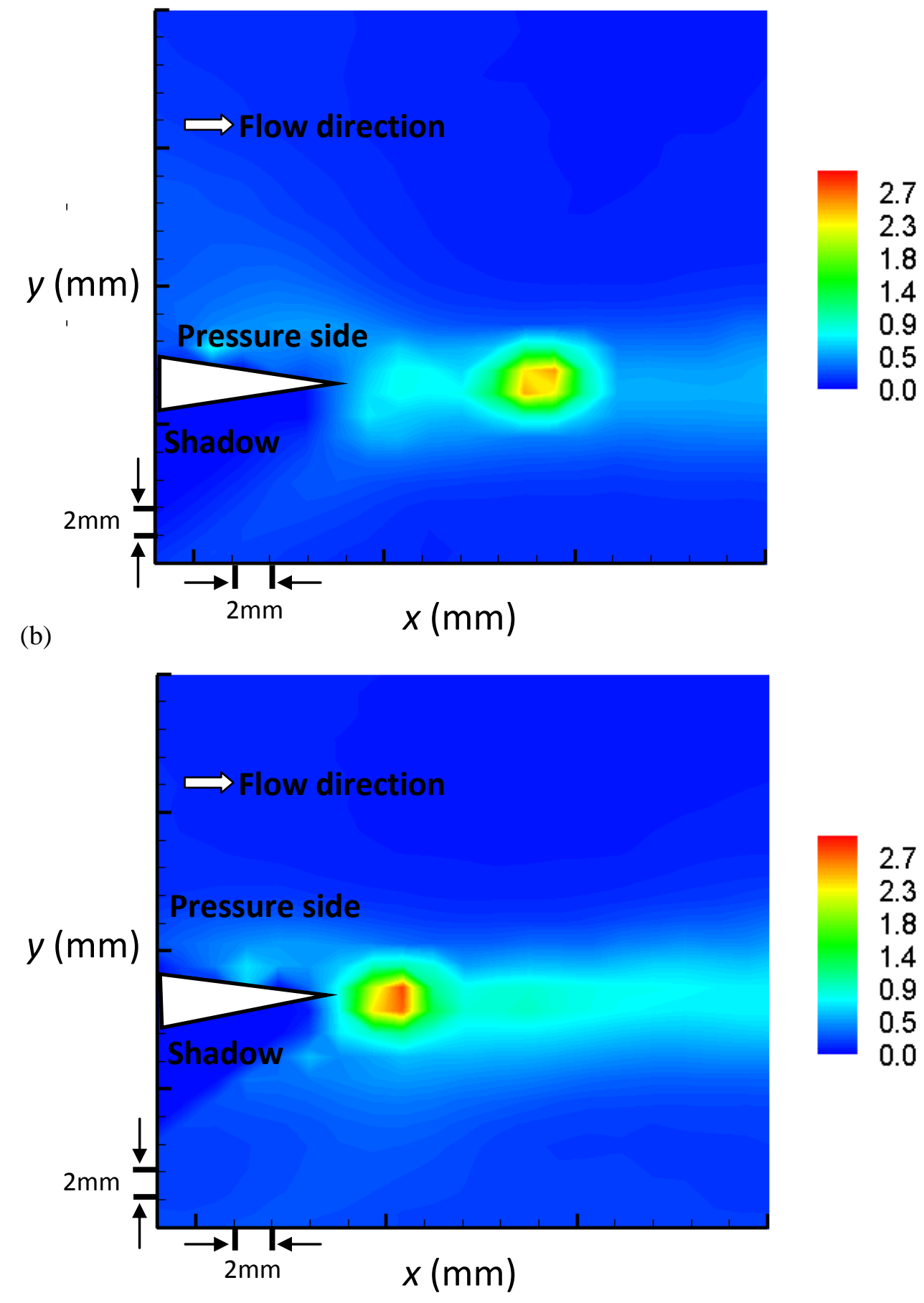

Fig. 22 Contour maps of the measured rms normal-component velocity fluctuation, $v_{\mathrm{rms}}\left(\mathrm{ms}^{-1}\right)$ for the $\mathrm{S} 0$ straight trailing edge at (a) $\theta=$ $0^{\circ}$; and (b) $\theta=2^{\circ}$. The freestream velocity is set at $15.9 \mathrm{~ms}^{-1}$. 
(a)

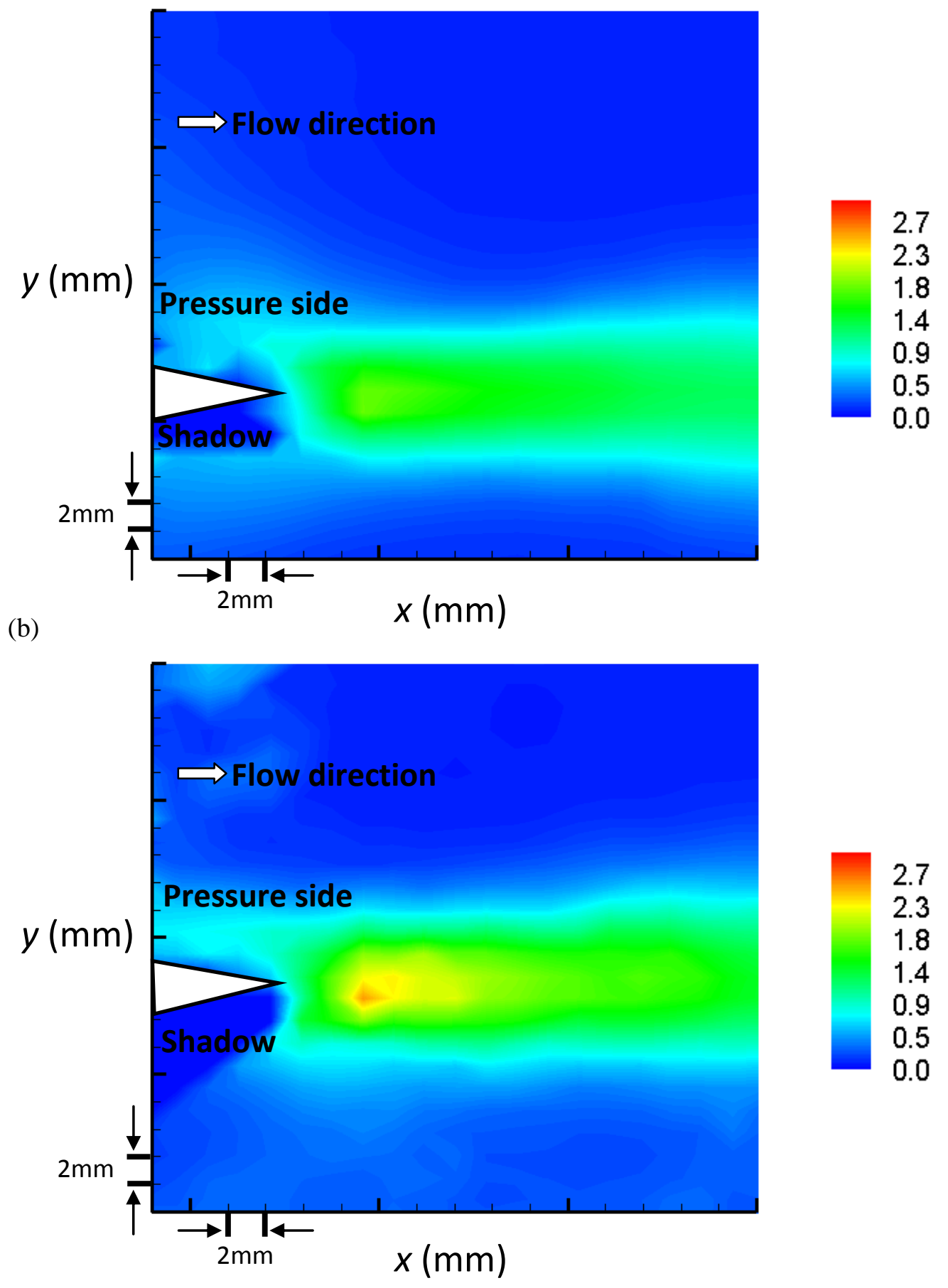

Fig. 23 Contour maps of the measured rms normal-component velocity fluctuation, $v_{\text {rms }}\left(\mathrm{ms}^{-1}\right)$ for the $\mathrm{S} 2$ serrated trailing edge at (a) $\theta$ $=0^{\circ}$; and (b) $\theta=2^{\circ}$. The freestream velocity is set at $15.9 \mathrm{~ms}^{-1}$. 


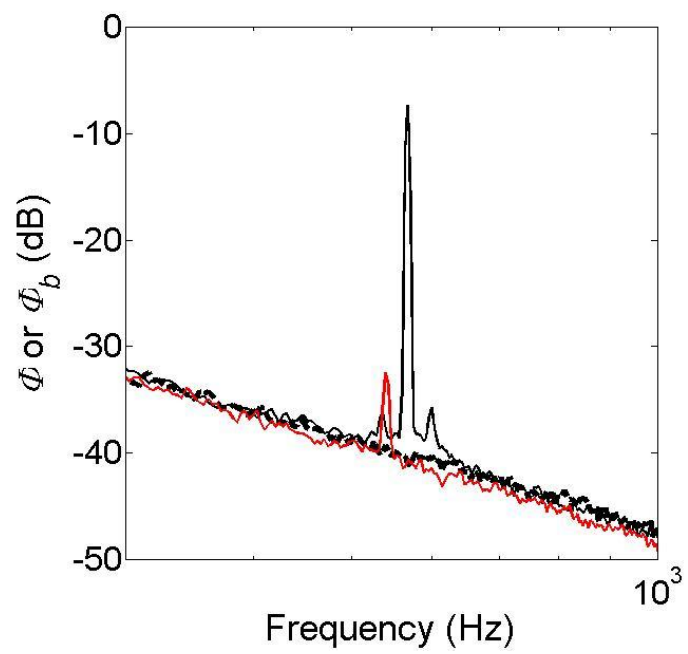

Fig. 24 Comparison of the airfoil noise spectra $\Phi$ measured at $\theta=0^{\circ}$ for SO (black -) and S2 (red -) trailing edges as well as the tunnel background noise $\Phi_{\mathrm{b}}(---)$. The freestream velocity is set at $15.9 \mathrm{~ms}^{-1}$. 


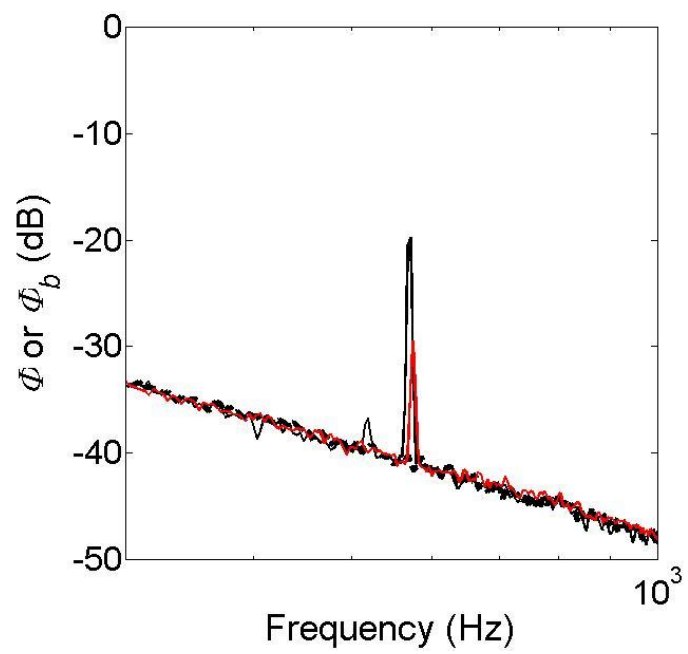

Fig. 25 Comparison of the airfoil noise spectra $\Phi$ measured at $\theta=2^{\circ}$ for S0 (black -) and S2 (red -) trailing edges as well as the tunnel background noise $\Phi_{\mathrm{b}}(---)$. The freestream velocity is set at $15.9 \mathrm{~ms}^{-1}$. 


\begin{tabular}{cccccc} 
& SO & & \multicolumn{3}{c}{ S2 } \\
$n$ & $f_{\mathrm{n}}$ (theory) & $f_{\mathrm{n}}($ exp. $)$ & $n$ & $f_{\mathrm{n}}$ (theory) & $f_{\mathrm{n}}($ exp. $)$ \\
$\mathrm{Hz}$ & $\mathrm{Hz}$ & & $\mathrm{Hz}$ & $\mathrm{Hz}$ \\
\hline 7 & 411 & & 7 & 470 & \\
8 & 475 & & 8 & 541 & $540\left(f_{\mathrm{n}-\max }\right)$ \\
9 & 541 & 539 & 9 & 611 & \\
& & $572\left(f_{\mathrm{n}-\max }\right)$ & & & \\
10 & 609 & 604 & 10 & 682 & \\
11 & 678 & & 11 & 752 &
\end{tabular}

Table 1 Summary of the measured discrete tone frequencies, $f_{\mathrm{n}}(\exp$.$) at \theta=$ $0^{\circ}$ for SO and S2 trailing edges measured in the closed-working section wind tunnel associated with the PIV experiment. The inlet freestream velocity was set at $15.9 \mathrm{~ms}^{-1}$. The theoretical discrete tone frequencies, $f_{\mathrm{n}}$ (theory) based on feedback Model A are presented. 


\begin{tabular}{cccccc} 
& SO & & \multicolumn{3}{c}{$\mathrm{S} 2$} \\
$n$ & $f_{\mathrm{n}}$ (theory) & $f_{\mathrm{n}}$ (exp.) & $n$ & $\begin{array}{c}f_{\mathrm{n}} \text { (theory) } \\
\mathrm{Hz}\end{array}$ & $\begin{array}{c}f_{\mathrm{n}}(\text { exp.) } \\
\mathrm{Hz}\end{array}$ \\
\hline 6 & 386 & & & 489 & \\
7 & 452 & & 7 & 568 & 575 \\
8 & 516 & 519 & 8 & 646 & \\
9 & 579 & 572 & 9 & 727 & \\
10 & 643 & & 10 & 812 &
\end{tabular}

Table 2 Summary of the measured discrete tone frequencies, $f_{\mathrm{n}}(\exp$.) at $\theta=$ $2^{\circ}$ for S0 and S2 trailing edges measured in the closed-working section wind tunnel associated with the PIV experiment. The inlet freestream velocity was set at $15.9 \mathrm{~ms}^{-1}$. The theoretical discrete tone frequencies, $f_{\mathrm{n}}$ (theory) based on feedback Model A are presented. 